TRANSACTIONS OF THE

AMERICAN MATHEMATICAL SOCIETY

Volume 356, Number 3, Pages 953-985

S 0002-9947(03)03409-3

Article electronically published on August 21, 2003

\title{
ANALYTIC ORDER OF SINGULAR AND CRITICAL POINTS
}

\author{
EUGENII SHUSTIN
}

\begin{abstract}
We deal with the following closely related problems: (i) For a germ of a reduced plane analytic curve, what is the minimal degree of an algebraic curve with a singular point analytically equivalent (isomorphic) to the given one? (ii) For a germ of a holomorphic function in two variables with an isolated critical point, what is the minimal degree of a polynomial, equivalent to the given function up to a local holomorphic coordinate change? Classically known estimates for such a degree $d$ in these questions are $\sqrt{\mu}+1 \leq d \leq \mu+1$, where $\mu$ is the Milnor number. Our result in both the problems is $d \leq a \sqrt{\mu}$ with an absolute constant $a$. As a corollary, we obtain asymptotically proper sufficient conditions for the existence of algebraic curves with prescribed singularities on smooth algebraic surfaces.
\end{abstract}

\section{INTRODUCTION}

We work with algebraic curves, hypersurfaces, and functions over the complex field $\mathbb{C}$, although main results hold for any algebraically closed field of characteristic 0 by the Lefschetz principle.

Statement of the problem and formulation of main results. A classical question is how many and what types of singular points can occur on a plane algebraic curve of a given degree. The well-known bound

$$
n \leq \frac{(d-1)(d-2)}{2}
$$

is necessary and sufficient for the existence of an irreducible plane curve of degree $d$ with $n$ nodes [18]. Even the case of cusps appears to be much more difficult (see [10, 11, 19]), so that one cannot expect a complete answer. However, we can ask for reasonable sufficient existence conditions which cover arbitrary degrees and singularities. Namely, the inequalities

$$
\mu_{0} \leq(d-1)^{2}, \quad \delta_{0} \leq \frac{(d-1)(d-2)}{2}
$$

are necessary for the existence of an irreducible plane curve of degree $d$ with given singularities having the total Milnor number $\mu_{0}$ and the total $\delta$-invariant $\delta_{0}$. The

Received by the editors July 5, 2002.

2000 Mathematics Subject Classification. Primary 14F17, 14H20; Secondary 58K05.

The author was partially supported by Grant No. G-616-15.6/99 of the German-Israeli Foundation for Research and Development and by the Hermann-Minkowski Minerva Center for Geometry at Tel Aviv University. This work was completed during the author's RiP stay at the Mathematisches Forschunsinstitut Oberwolfach. 
main result of [6] (refined later in [14]) states that the condition

$$
\mu_{0} \leq \frac{1}{46}(d+2)^{2}
$$

is sufficient for the existence of an irreducible plane curve of degree $d$ with given singularities prescribed up to topological equivalence. Asymptotically it coincides with the necessary condition up to a constant factor, and, thus, is called asymptotically proper.

However, (1) does not apply to singularities defined up to analytic equivalence 1 Our first result (Theorem 3 and Remark 5 section 3.1) is that the inequality

$$
\mu_{0} \leq \frac{1}{9}\left(d^{2}-2 d+3\right)
$$

is sufficient for the existence of an irreducible plane curve of degree $d$ with arbitrary singularities prescribed up to analytic equivalence and with the total Milnor number $\mu_{0}$. This not only covers a wider range than (1), but is concerned with a stronger equivalence relation for singular points. In the case of one singularity represented by a curve germ $(C, z)$, we estimate the analytic order $d(C, z)$ of this germ, i.e., the minimal degree of a plane curve having a singular point analytically equivalent to $(C, z)$, as

$$
d(C, z) \leq 3 \sqrt{\mu(C, z)}-1
$$

(Theorem 2 and Remark [5, section 3.1). A closely related question: given a germ of a holomorphic function $f:\left(\mathbb{C}^{2}, 0\right) \rightarrow(\mathbb{C}, 0)$ with an isolated critical point, what is the analytic order $d(f)$ of this germ, i.e., the minimal degree of a polynomial equivalent to $f$ up to a local holomorphic coordinate change (so-called right equivalence)? We refine the classical bounds

$$
\sqrt{\mu(f)}+1 \leq d(f) \leq \mu(f)+1
$$

with our upper bound

$$
d(f)<4 \sqrt{\mu(f)}-1
$$

(Theorem [5] section 4).

These questions can be generalized in two directions. First, one can look for curves with prescribed singularities in given linear systems on smooth algebraic surfaces. We provide a sufficient numerical condition for the existence of an irreducible curve with singularities prescribed up to analytic equivalence in a given linear system on a smooth algebraic surface (Theorem 4, section [3.4). It is stronger than a similar sufficient existence condition which was found in [13] and concerned only the topological equivalence of singular points. Another way is a higher-dimensional generalization. For example, for holomorphic function germs $f:\left(\mathbb{C}^{n}, 0\right) \rightarrow(\mathbb{C}, 0)$, it is known that

$$
\sqrt[n]{\mu(f)}+1 \leq d(f) \leq \mu(f)+1 .
$$

We conjecture that

$$
d(f) \leq a_{n} \sqrt[n]{\mu(f)}
$$

with $a_{n}>0$ depending only on $n$, and we prove this for germs of type $A_{k}, k \geq 1$ (Theorem [6] section 5).

\footnotetext{
${ }^{1}$ Also called analytic isomorphism or contact equivalence.
} 
Idea of the proof. Similarly to [6, 21] we introduce certain zero-dimensional schemes $Z \subset \mathbb{P}^{2}$ associated with singular and critical points, whose degree (length) is bounded by a linear function of the (total) Milnor number, and such that, for

$$
d=\min \left\{n \geq 1: H^{1}\left(\mathbb{P}^{2}, \mathcal{J}_{Z}(n)\right)=0\right\}, \quad \mathcal{J}_{Z}=\operatorname{Ker}\left(\hat{\mathcal{O}}_{\mathbb{P}^{2}} \rightarrow \hat{\mathcal{O}}_{Z}\right)
$$

there is a curve (polynomial) of degree $d$ with singular (critical) points of given types (Lemmas $3(2)$, section 1.2 Lemma 5 , section 1.3 and proof of Theorem 5 section (4). In principle, $d$ may be as large as $\operatorname{deg} Z-1$. We, however, can choose $Z$ to be generic in $\operatorname{Iso}(Z)$, the set of zero-dimensional schemes isomorphic to $Z$ as subschemes of $\mathbb{P}^{2}$, and then establish our principal bound (Proposition 10, section 2.1)

$$
d<\frac{4}{\sqrt{3}} \sqrt{\operatorname{deg} Z}-2 \quad \text { as } \quad \operatorname{deg} Z>2,
$$

which provides the main estimates for the analytic order of a singular or critical point.

In [6 an upper bound like (2) is obtained for irreducible zero-dimensional schemes of cluster type, generic in their deformation class (which can be rather larger than the isomorphism class). The proof was based on the so-called "Horace method" suggested by Hirschowitz [12]. It consists of an inductive procedure, where on each step one specializes a zero-dimensional scheme (in its deformation class) on a given line, then passes to the residue scheme. However, this approach fails in our situation. The main obstacle (besides many technical ones) is that, starting with a zero-dimensional scheme $Z$ generic in $\operatorname{Iso}(Z)$, we have to specialize it in a certain way, and then obtain a residue scheme which is no longer generic in its isomorphism class; thus, the induction assumption does not apply.

To obtain (2), we exploit a different idea, which is similar in a sense to that in [25, where $h^{1}$-vanishing for some zero-dimensional schemes in the plane is deduced from the ampleness of some divisors of the blown-up plane by Kodaira's theorem. Namely, we start with estimating the minimal degree of a curve, containing a scheme $Z$ generic in Iso $Z$ ), from below by $\sqrt{\operatorname{deg} Z} / 2$ (Proposition 8 , section 2.1). For, we observe that, deforming $Z$ in $\operatorname{Iso}(Z)$ so that the curve of minimal degree through the scheme changes, one obtains that either an intersection of $Z$ with a close element of $\operatorname{Iso}(Z)$ is of a (relatively) large length, or $Z$ determines a singularity with large invariants so that the curves through close schemes have many intersections in neighborhood of singularities, and then the desired estimate comes from Bézout's theorem. A combination of such arguments can be found in 25] when zero-dimensional schemes define ordinary singular points; in the general case we use estimates from [8]. Next, instead of exploring ampleness which does not seem to be easy to apply in our problem, we use the Castelnuovo function theory (see [3, 7]). The latter argument appears to be quite simple and transparent. The graph of the (positive) Castelnuovo function of a zero-dimensional scheme $Z$ has width $d+1$, where $d$ is from (2), its height equals the minimal degree of a curve through $Z$, which is $\sim \sqrt{\operatorname{deg} Z}$, and the area of its convex hull is $\operatorname{deg} Z$. Thus, one obtains $d \sim \sqrt{\operatorname{deg} Z}$ when getting rid of long horizontal segments of the graph. The latter can be done by Davis' lemma [3] (see details in the proof of Proposition 10. section [2.1] and in [7]). 
Finally, we notice that one could similarly treat zero-dimensional schemes $Z \subset$ $\mathbb{P}^{n}, n \geq 3$. Indeed, it is not difficult to show that the minimal degree of a hypersur-

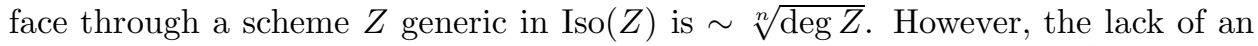
appropriate Castelnuovo function theory prevents making the step to $h^{1}$-vanishing bounds.

Remark 1. One may ask what is the minimal possible coefficient of $\sqrt{\operatorname{deg} Z}$ in (2). An example of two "fat" 2 points of equal multiplicities shows that it cannot be less than 2 , which is close to our value $4 / \sqrt{3}=2.30 \ldots$.

The Harbourne-Hirschowitz conjecture [12] (see a survey and bibliography in [2]) states that, for the scheme $Z$ of "fat" points in general position in the plane, $h^{1}\left(\mathbb{P}^{2}, \mathcal{J}_{Z}(d)\right)=0$ as far as $h^{0}\left(\mathbb{P}^{2}, \mathcal{J}_{Z}(d)\right) \geq 0$ and $d$ is greater than or equal to the sum of any three multiplicities, i.e., $d \approx \sqrt{2 \operatorname{deg} Z}$ in (2). It is not clear what should be an analogue of this conjecture for arbitrary schemes if any. A reasonable conjecture can be an analogue of the Alexander-Hirschowitz theorem [1: for any $k \geq 1$ there exists $N(k) \geq 1$ such that $h^{1}\left(\mathbb{P}^{2}, \mathcal{J}_{Z}(d)\right)=0$, provided $d \geq N(k)$ and $h^{0}\left(\mathbb{P}^{2}, \mathcal{J}_{Z}(d)\right) \geq 0$, for any scheme $Z \subset \mathbb{P}^{2}$ with irreducible components of length $\leq k$ and which is generic in $\operatorname{Iso}(Z)$.

\section{ZERO-DIMENSIONAL SCHEMES ASSOCIATED WITH SINGULAR AND CRITICAL POINTS}

1.1. Zero-dimensional schemes: Cluster schemes, numerical invariants, deformation and isomorphism classes. Throughout the paper, we work with zero-dimensional schemes $Z$ that are contained in a smooth algebraic surface $\Sigma$. The corresponding ideal sheaves will be denoted by $\mathcal{J}_{Z / \Sigma} \subset \hat{\mathcal{O}}_{\Sigma}$. Moreover, we denote

$$
\operatorname{deg} Z=\sum_{z} \operatorname{dim}_{\mathbb{C}} \hat{\mathcal{O}}_{\Sigma, z} /\left(\mathcal{J}_{Z / \Sigma}\right)_{z}, \quad \operatorname{mt}(Z, z)=\max \left\{n \in \mathbb{Z}:\left(\mathcal{J}_{Z / \Sigma}\right)_{z} \subset \mathfrak{m}_{z}^{n}\right\},
$$

with $\hat{\mathcal{O}}_{\Sigma, z}$ the analytic local ring at $z$ and $\mathfrak{m}_{z} \subset \hat{\mathcal{O}}_{\Sigma, z}$ the maximal ideal.

Let $z$ be an isolated singular point of an algebraic curve $C \subset \Sigma$. Denote by $T^{\infty}(C, z)$ the complete (infinite) embedded resolution tree of the singular point $(C, z)$. The root vertex of $T^{\infty}(C, z)$ is $z$. Among the other vertices of $T^{\infty}(C, z)$ there are finitely many which are not nodes of the union of the exceptional locus with the corresponding strict transform of $(C, z)$. All these vertices of $T^{\infty}(C, z)$ together with $z$ are called essential infinitely near points of $(C, z)$. They are vertices of a subtree $T^{*}(C, z) \subset T^{\infty}(C, z)$.

Define the multiplicity of $C$ at $z$ as

$$
\operatorname{mt}(C, z)=\max \left\{n \geq 0: C \in \mathfrak{m}_{z}^{n}\right\} .
$$

Correspondingly, for any vertex $q \in T^{\infty}(C, z)$, by the multiplicity $\operatorname{mt}(C, q)$ of $C$ at $q$ we mean the multiplicity of the respective strict transform of $C$ at $q$. For example,

$$
\operatorname{mt}(C, q)=1, \quad q \in T^{\infty}(C, z) \backslash T^{*}(C, z) .
$$

Given a finite subtree $T \subset T^{\infty}(C, z)$ such that $T \supset T^{*}(C, z)$, we define a zerodimensional cluster scheme $Z=Z(T)$ by the ideal $I(Z) \subset \hat{\mathcal{O}}_{\Sigma, z}$ generated by all

\footnotetext{
${ }^{2}$ That is, defined by powers of the maximal ideals.
} 
germs $f \in \hat{\mathcal{O}}_{\Sigma, z}$ satisfying

$$
\operatorname{mt}(f, q)=\operatorname{mt}(C, q) \stackrel{\text { def }}{=} \operatorname{mt}(Z, q), \quad q \in T .
$$

Such schemes were introduced in [6] as "generalized singularity schemes". The above generators $f$ of the ideal $I(Z)$ are called generic elements of $I(Z)$.

Lemma 1 ([6], Lemma 2.4). (1) The vertices of $T=T(Z)$ form the base point set of the ideal $I(Z)$.

(2) Almost all germs $f \in I(Z)$ are generic.

A reducible zero-dimensional scheme $Z \subset \Sigma$, concentrated at points $z_{1}, \ldots, z_{p}$, whose irreducible components $Z_{z_{1}}, \ldots, Z_{z_{p}}$ are cluster schemes, is called a cluster scheme as well, and $T(Z)$ is defined as the disjoint union of the trees $T\left(Z_{z_{i}}\right)$, $i=1, \ldots, p$.

Let $Z$ be an arbitrary irreducible zero-dimensional scheme in $\mathbb{P}^{2}$. There exists a unique maximal cluster subscheme $Z_{c l} \subset Z$. Namely, $T\left(Z_{c l}\right)$ is the tree of infinitely near base points of the ideal $I(Z)$, and $\operatorname{mt}\left(Z_{c l}, q\right), q \in T\left(Z_{c l}\right)$, are the corresponding multiplicities of a generic element of $I(Z)$; more precisely, a generic element in the linear space spanned by (finitely many) generators of $I(Z)$. If $Z$ is reducible, then $Z_{c l}$ is the union of the maximal cluster subschemes of the components of $Z$.

Put

$$
M_{2}(Z)=\sum_{q \in T\left(Z_{c l}\right)}\left(\operatorname{mt}\left(Z_{c l}, q\right)\right)^{2}
$$

We claim that

$$
\operatorname{deg} Z \leq M_{2}(Z)=\operatorname{deg} Z_{c l}+\delta,
$$

where $\delta$ is the $\delta$-invariant of a generic member of $I(Z)$. The equality in (3) follows from the formulas in [6], Lemma 2.6. For the inequality suppose that $Z$ is concentrated at point $z$. Then take two distinct generic elements $f, g \in I(Z)$, and obtain

$$
Z \subset f \cap g \quad \Longrightarrow \quad \operatorname{deg} Z \leq(f \cdot g)_{z}=\sum_{q \in T\left(Z_{c l}\right)}\left(\operatorname{mt}\left(Z_{c l}, q\right)\right)^{2}=M_{2}(Z) .
$$

Note that (3) implies

$$
M_{2}(Z)<2 \cdot \operatorname{deg} Z
$$

For a zero-dimensional scheme $Z$ with $p$ irreducible components, its isomorphism class Iso $(Z)$ is fibred over the space of $p$-tuples $\left(z_{1}, \ldots, z_{p}\right) \in\left(\mathbb{P}^{2}\right)^{p}$ with fibre being an orbit of the action of the group $\prod_{i=1}^{p} \operatorname{Aut}\left(\hat{\mathcal{O}}_{\mathbb{P}^{2}, z_{i}} / \mathfrak{m}_{z_{i}}^{n}\right)$, where $n$ is sufficiently large. If $Z$ is a cluster scheme, then one can naturally define the set $\operatorname{Def}(Z)$ of schemes $Z^{\prime} \subset \mathbb{P}^{2}$, which are deformation equivalent to $Z$. This is a smooth irreducible quasiprojective variety [7], section 2. Clearly, $\operatorname{Def}(Z) \supset \operatorname{Iso}(Z)$.

A zero-dimensional scheme is called nonsingular, if the generic elements of the ideals of its components are nonsingular, and is called singular otherwise. We notice that a nonsingular zero-dimensional scheme is always a cluster scheme. 
1.2. Zero-dimensional schemes associated with topological types of singular points. Let $z$ be an isolated singular point of a curve $C \subset \Sigma$. The equisingularity ideal introduced in [23] (see also [4, 5, 6, 7] 20]) is defined as

$$
I^{e s}(C, z):=\left\{g \in \hat{\mathcal{O}}_{\Sigma, z}: f+\varepsilon g \text { is equisingular over } \operatorname{Spec}\left(\mathbb{C}[\varepsilon] / \varepsilon^{2}\right)\right\},
$$

where $f \in \hat{\mathcal{O}}_{\Sigma, z}$ is a germ induced by $C$. The zero-dimensional scheme defined by $I^{e s}(C, z)$ is denoted by $Z^{e s}(C, z)$.

The notion of the cluster scheme directly relates to the topological equivalence of germs. Namely, 6], Lemma 2.4, implies

Lemma 2. For any irreducible deformation equivalent cluster schemes $Z$ and $Z^{\prime}$, generic elements $f \in I(Z), f^{\prime} \in I\left(Z^{\prime}\right)$ are topologically equivalent.

Let $(C, z) \subset\left(\mathbb{P}^{2}, z\right)$ be a reduced curve germ. We associate two zero-dimensional schemes with it (cf. [6]):

- $Z^{s}(C, z)$, the cluster scheme defined by the germ $(C, z)$ and the tree of essential point $T^{*}(C, z)$;

- $Z_{1}^{s}(C, z)=Z^{s}(\widetilde{C}, z)$, the cluster scheme defined by the germ $(L C, z)$, where $L$ is a straight line through $z$ and transverse to $C$.

The importance of these schemes arises from

Lemma 3. (1) The scheme $Z^{s}(C, z)$ is minimal among the zero-dimensional schemes $Z$ such that almost all germs $f \in I(Z)$ are topologically equivalent to $(C, z)$.

(2) Let some zero-dimensional scheme $Z$ such that $z \notin Z$, satisfy

$$
H^{1}\left(\mathcal{J}_{Z_{1}^{s}(C, z) \cup Z / \mathbb{P}^{2}}(d)\right)=0 .
$$

Then there exist $D \in\left|\mathcal{J}_{Z^{s}(C, z) \cup Z / \mathbb{P}^{2}}(d)\right|$ such that $(D, z)$ is topologically equivalent to $(C, z)$. Moreover, these curves $D$ form a dense open subset in $\left|\mathcal{J}_{Z^{s}(C, z) \cup Z / \mathbb{P}^{2}}(d)\right|$.

Clearly $Z^{s}(C, z) \supset Z^{e s}(C, z)$. Moreover, it can be shown that $Z^{s}(C, z)$ is the minimal cluster scheme containing $Z^{e s}(C, z)$.

Proof. The first statement reflects the fact that the tree of essential infinitely near points of $(C, z)$ and multiplicities of $C$ at them (uniquely) determine the topological type of $(C, z)$.

The second statement can be proven in the same way as it is done in Step 1 of the proof of Lemma 5.8 in [6], where $Z$ is supposed to be empty, $Z_{1}^{S}(C, z)$ is denoted by $\widetilde{X}$, and the required $h^{1}$-vanishing condition is found in (5.12). Note only that the scheme $X^{\prime}$, used in this proof, is a subscheme of $\widetilde{X}$; hence the $h^{1}$-vanishing for $X^{\prime}$ mentioned in (5.12) follows from that for $\widetilde{X}=Z_{1}^{s}(C, z)$.

1.3. Zero-dimensional schemes associated with analytic types of singular points. In the previous notation, we introduce the zero-dimensional scheme $Z^{e a}(C, z)$ defined by the Tjurina ideal

$$
I^{e a}(C, z):=\left\langle f, f_{x}, f_{y}\right\rangle \subset \hat{\mathcal{O}}_{\Sigma, z},
$$

where $f(x, y)=0$ is a local equation for the germ $(C, z)$. The ideal $I^{e a}(C, z)$ is the tangent space to equianalytic (i.e., analytically trivial) deformations of $(C, z)$.

For our purpose we shall use zero-dimensional schemes associated with analytic types of singular points, which are analogous to $Z^{s}, Z_{1}^{s}$, but without the minimality 
property as in Lemma 31) (except for the simple singularities $A_{k}, k \geq 1, D_{k}$, $k \geq 4, E_{6}, E_{7}, E_{8}$, for which topological and analytic equivalence coincide).

If the singular point $z$ of $C$ is simple, we put

$$
Z^{a}(C, z)=Z^{s}(C, z), \quad Z_{1}^{a}(C, z)=Z_{1}^{s}(C, z) .
$$

If the singular point $z$ of $C$ is not simple, $f(x, y)=0$ is an equation of $C$ in a neighborhood of $z$, following [7], section 1.3, and we define

- the zero-dimensional scheme $Z^{a}(C, z)$ by the ideal

$$
I^{a}(C, z)=\left\{g \in \hat{\mathcal{O}}_{\mathbb{P}^{2}, z}:\left\langle g, g_{x}, g_{y}\right\rangle \subset\left\langle f, f_{x}, f_{y}\right\rangle\right\},
$$

- the zero-dimensional scheme $Z_{1}^{a}(C, z)$ by the ideal $I_{1}^{a}(C, z)=\mathfrak{m}_{z} \cdot I^{a}(C, z)$.

Observe that $Z_{1}^{a}(C, z) \supset Z^{a}(C, z) \supset Z^{e a}(C, z) \supset Z^{e s}(C, z)$.

Lemma 4. In the above notation,

(1) any scheme $Z \in \operatorname{Iso}\left(Z^{a}(C, z)\right)$ (resp., $Z \in \operatorname{Iso}\left(Z_{1}^{a}(C, z)\right)$ ) is $Z^{a}(\widetilde{C}, w)$ (resp., $\left.Z_{1}^{a}(\widetilde{C}, w)\right)$ for some germ $(\widetilde{C}, w)$ analytically equivalent to $(C, z)$;

(2) if $g \in I^{a}(C, z)$ (resp., $g \in I_{1}^{a}(C, z)$ ), then, for almost all $t \in \mathbb{C}$ (resp., for all $t \in \mathbb{C})$ the curve germs $\{f=0\}$ and $\{f+t g=0\}$ are analytically equivalent.

Proof. The first statement is evident. The fact that, for $g \in I^{a}(C, z)$ and almost all $t \in \mathbb{C}$, the germs $\{f=0\}$ and $\{f+t g=0\}$ are analytically equivalent, follows from the Mather-Yau theorem [16] (see also Lemma 1.8(a) of [7).

Assume now that $g \in I_{1}^{a}(C, z)=\mathfrak{m}_{z} \cdot I^{a}(C, z)$. Observe that if $h \in I^{a}(C, z)$, then $h=a f+b f_{x}+c f_{y}, b, c \in \mathfrak{m}_{z}$, which follows from Lemma 1.8(a,c) of [7]. Then

$$
\begin{gathered}
g=a f+\left(x b^{\prime}+y b^{\prime \prime}\right) f_{x}+\left(x c^{\prime}+y c^{\prime \prime}\right) f_{y}, \quad a, b^{\prime}, b^{\prime \prime}, c^{\prime}, c^{\prime \prime} \in \mathfrak{m}_{z}, \\
b^{\prime} f_{x x}+c^{\prime} f_{x y}, b^{\prime \prime} f_{x x}+c^{\prime \prime} f_{x y}, b^{\prime} f_{x y}+c^{\prime} f_{y y}, b^{\prime} f_{x y}+c^{\prime} f_{y y} \in\left\langle f, f_{x}, f_{y}\right\rangle .
\end{gathered}
$$

Since $1+a \in \hat{\mathcal{O}}_{\mathbb{P}^{2}, z}^{*}$, the germ $f+g=f+a f+\left(x b^{\prime}+y b^{\prime \prime}\right) f_{x}+\left(x c^{\prime}+y c^{\prime \prime}\right) f_{y}$ is equivalent to $f+\widetilde{g}$,

$$
\widetilde{g}=\frac{x b^{\prime}+y b^{\prime \prime}}{1+a} f_{x}+\frac{x c^{\prime}+y c^{\prime \prime}}{1+a} f_{y} .
$$

The restrictions to $b^{\prime}, b^{\prime \prime}, c^{\prime}, c^{\prime \prime}$ in (6), (17) yield that

$$
\left\langle\widetilde{g}, \widetilde{g}_{x}, \widetilde{g}_{y}\right\rangle \subset \mathfrak{m}_{z} \cdot\left\langle f, f_{x}, f_{y}\right\rangle,
$$

so the equivalence of $f$ and $f+\widetilde{g}$ follows from the Mather-Yau theorem [16] (cf. Lemma 1.8(b) of [7])

Lemma 5. Let a zero-dimensional scheme $Z$ such that $z \notin Z$ satisfy

$$
H^{1}\left(\mathcal{J}_{Z_{1}^{a}(C, z) \cup Z / \mathbb{P}^{2}}(d)\right)=0 .
$$

Then there exists a curve $D \in\left|\mathcal{J}_{Z^{a}(C, z) \cup Z / \mathbb{P}^{2}}(d)\right|$, whose germ $(D, z)$ is analytically equivalent to $(C, z)$. Moreover, such curves $D$ form a dense open subset in $\left|\mathcal{J}_{Z^{a}(C, z) \cup Z / \mathbb{P}^{2}}(d)\right|$.

Proof. In the exact sequence

$$
H^{0}\left(\mathcal{J}_{Z / \mathbb{P}^{2}}(d)\right) \rightarrow \hat{\mathcal{O}}_{\mathbb{P}^{2}, z} / I_{1}^{a}(C, z) \rightarrow H^{1}\left(\mathcal{J}_{Z \cup Z_{1}^{a}(C, z) / \mathbb{P}^{2}}(d)\right)=0,
$$

the first morphism is surjective. Denote by $\varphi \in \hat{\mathcal{O}}_{\mathbb{P}^{2}, z} / I_{1}^{a}(C, z)$ the image of a germ $\psi \in \hat{\mathcal{O}}_{\mathbb{P}^{2}, z}$ defined by $(C, z)$. Take $\Phi \in H^{0}\left(\mathcal{J}_{Z / \mathbb{P}^{2}}(d)\right)$ which projects to $\varphi$. Then $\Phi-\psi \in I_{1}^{a}(C, z)$, and by Lemma $4(2)$, the curve germs $(\{\Phi=0\}, z)$ and $(C, z)$ are analytically equivalent, thus, we can put $D=\{\Phi=0\}$. 
1.4. Zero-dimensional schemes associated with analytic types of critical points. Let $f:\left(\mathbb{C}^{2}, 0\right) \rightarrow(\mathbb{C}, 0)$ be a germ of a holomorphic function with a finite Milnor number $\mu(f)=\operatorname{dim} \hat{\mathcal{O}}_{\mathbb{C}^{2}, 0} /\left\langle f_{x}, f_{y}\right\rangle$. Germs $f, g \in \hat{\mathcal{O}}_{\mathbb{C}^{2}, 0}$ are called (right) equivalent, if there is $\psi \in \operatorname{Aut}\left(\hat{\mathcal{O}}_{\mathbb{C}^{2}, 0}\right)$ such that $g=f \circ \psi$. Introduce the zerodimensional schemes

- $Z_{0}(f)$ defined by the ideal

$$
I_{0}(f)=\left\{g \in \hat{\mathcal{O}}_{\mathbb{C}^{2}, 0}: g, g_{x}, g_{y} \in\left\langle f_{x}, f_{y}\right\rangle\right\} ;
$$

- $Z(f)$ defined by the ideal $I(f)=\mathfrak{m}_{0} I_{0}(f)$.

An analogue of Lemma 4 reads as

Lemma 6. In the above notation,

(1) any scheme $Z \in \operatorname{Iso}(Z(f))$ is $Z(\tilde{f})$ for some germ $\tilde{f}$ equivalent to $f$;

(2) if $g \in I(f)$, then, for all $t \in \mathbb{C}$, the germ $f+t g$ is equivalent to $f$.

The first statement of the lemma is evident. The second one is, in fact, known and can be proven as Mather's finite determinacy theorem [15].

1.5. Bounds for degrees of zero-dimensional schemes. Given a reduced curve germ $(C, z) \subset \mathbb{P}^{2}$ of a function germ $f \in \hat{\mathcal{O}}_{\mathbb{C}^{2}, 0}$, the degrees of the schemes $Z^{s}(C, z)$, $Z^{a}(C, z), Z(f)$ are invariants of the given singular or critical point up to the corresponding equivalence. We shall compare these invariants with the classical ones.

Lemma 7. In the above notation,

$$
\operatorname{deg} Z^{s}(C, z)=\operatorname{deg} Z^{a}(C, z)=\operatorname{deg} Z_{0}(f)=\left[\frac{3 k+4}{2}\right],
$$

if $(C, z)$ or $f \in \hat{\mathcal{O}}_{\mathbb{C}^{2}, 0}$ is of type $A_{k}, k \geq 1$. For other singular and critical points

$$
\begin{gathered}
\operatorname{deg} Z^{s}(C, z) \leq 3 \delta(C, z), \\
\operatorname{deg} Z^{a}(C, z) \leq 2 \mu(C, z), \\
\operatorname{deg} Z_{0}(f) \leq 3 \mu(f)-2 \cdot \operatorname{mt}(f)+2 .
\end{gathered}
$$

Proof. Formula (9) is an easy computation along the definition of $Z^{s}(C, z)=$ $Z^{a}(C, z)=Z_{0}(f)$ in this case.

Assume that $\operatorname{mt}(C, z) \geq 3$. Then

$$
\operatorname{deg} Z^{s}(C, z)=\sum_{q \in T^{*}(C, z)} \frac{\operatorname{mt}(C, q) \cdot(\operatorname{mt}(C, q)+1)}{2}=\delta(C, z)+\sum_{q \in T^{*}(C, z)} \operatorname{mt}(C, q) .
$$

By definition of $T^{*}(C, z)$,

$$
\#\left\{q \in T^{*}(C, z): \operatorname{mt}(C, q)=1\right\} \leq \operatorname{mt}(C, z)=m .
$$

Hence

$$
\begin{aligned}
& \sum_{q \in T^{*}(C, z)} \operatorname{mt}(C, q) \leq 2 \cdot \operatorname{mt}(C, z)+\sum_{\substack{q \in T^{*}(C, z) \\
q \neq z \\
\operatorname{mt}(C, q)>1}} \operatorname{mt}(C, q) \\
& \leq 2 \sum_{q \in T^{*}(C, z)} \frac{\operatorname{mt}(C, z) \cdot(\operatorname{mt}(C, z)-1)}{2}=2 \delta(C, z),
\end{aligned}
$$

and (10) follows. 
We shall establish (11), first, for simple singularities $D_{k}, k \geq 4, E_{k}, k=6,7,8$. Here $Z^{a}(C, z)=Z^{s}(C, z)$, and a direct computation gives

$$
\begin{aligned}
& \operatorname{deg} Z^{a}\left(D_{k}\right)=\operatorname{deg} Z^{s}\left(D_{k}\right)=\left[\frac{3 k+1}{2}\right] \leq 2 k, \quad k \geq 4, \\
& \operatorname{deg} Z^{a}\left(E_{k}\right)=\operatorname{deg} Z^{s}\left(E_{k}\right)=k+3 \leq 2 k, \quad k=6,7,8 .
\end{aligned}
$$

If $(C, z)$ is not simple, introduce $\Pi_{1}, \Pi_{2}$, two distinct generic polar curves of $C$, and $\Pi_{11}, \Pi_{12}$, two generic polar curves of $\Pi_{1}$. By [7], formula (1.5),

$$
I^{a}(C, z) \supset\left\{f C+g \Pi_{1}: f, g \in \hat{\mathcal{O}}_{\mathbb{P}^{2}, z}, g \Pi_{11}, g \Pi_{12} \in\left\langle\Pi_{1}, \Pi_{2}\right\rangle\right\} .
$$

By the double point divisor theorem [22], $\S 50$, the ideal

$$
\left\{g \in \hat{\mathcal{O}}_{\mathbb{P}^{2}, z}: g \Pi_{11}, g \Pi_{12} \in\left\langle\Pi_{1}, \Pi_{2}\right\rangle\right\}
$$

contains the ideal

$$
\begin{gathered}
I=\left\{g \in \hat{\mathcal{O}}_{\mathbb{P}^{2}, z}:(g \cdot P)_{z} \geq\left(\Pi_{2} \cdot P\right)_{z}-\operatorname{mt}(P, z)+1\right. \\
\text { for any local branch } \left.P \text { of }\left(\Pi_{1}, z\right)\right\},
\end{gathered}
$$

where $(*, *)_{z}$ denotes the intersection multiplicity of two curve germs at the point $z$. 3 Here

$$
\begin{gathered}
\operatorname{dim} \hat{\mathcal{O}}_{\mathbb{P}^{2}, z} / I=\sum_{P}\left(\left(\Pi_{2} \cdot P\right)_{z}-\operatorname{mt}(P, z)+1\right)-\delta\left(\Pi_{1}, z\right) \\
\leq \sum_{P}\left(\Pi_{2} \cdot P\right)_{z}-\delta\left(\Pi_{1}, z\right)=\mu(C, z)-\delta\left(\Pi_{1}, z\right) .
\end{gathered}
$$

If $\operatorname{mt}(C, z)=3$ and $(C, z)$ is not simple, then $\Pi_{1}$ has at least a tacnode at $z$, so $\delta\left(\Pi_{1}, z\right) \geq 2=\operatorname{mt}(C, z)-1$. If $\operatorname{mt}(C, z) \geq 4$, then $\operatorname{mt}\left(\Pi_{1}, z\right)=\operatorname{mt}(C, z)-1 \geq 3$, so

$$
\delta\left(\Pi_{1}, z\right) \geq \frac{\operatorname{mt}\left(\Pi_{1}, z\right) \cdot\left(\operatorname{mt}\left(\Pi_{1}, z\right)-1\right)}{2} \geq \operatorname{mt}\left(\Pi_{1}, z\right)=\operatorname{mt}(C, z)-1,
$$

which altogether results in $\operatorname{dim} \hat{\mathcal{O}}_{\mathbb{P}^{2}, z} / I \leq \mu(C, z)-\operatorname{mt}(C, z)+1$. Hence

$$
\begin{gathered}
\operatorname{deg} Z^{a}(C, z)=\operatorname{dim} \hat{\mathcal{O}}_{\mathbb{P}^{2}, z} / I^{a}(C, z) \leq\left(C \cdot \Pi_{1}\right)_{z}+\operatorname{dim} \hat{\mathcal{O}}_{\mathbb{P}^{2}, z} / I \\
\leq(\mu(C, z)+\operatorname{mt}(C, z)-1)+(\mu(C, z)-\operatorname{mt}(C, z)+1)=2 \mu(C, z) .
\end{gathered}
$$

For inequality (12) we note that

$$
\begin{gathered}
I_{0}(f) \supset\left\{g \in \hat{\mathcal{O}}_{\mathbb{C}^{2}, 0}: g=a f_{x}+b f_{y}, a, b \in \hat{\mathcal{O}}_{\mathbb{C}^{2}, 0}\right. \\
\left.a f_{x x}, a f_{x y}, b f_{x y}, b f_{y y} \in\left\langle f_{x}, f_{y}\right\rangle\right\} \\
\supset\left\{g \in \hat{\mathcal{O}}_{\mathbb{C}^{2}, 0}: g=a f_{x}+b f_{y}, a, b \in I\right\},
\end{gathered}
$$

where the ideal $I \subset \hat{\mathcal{O}}_{\mathbb{C}^{2}, 0}$ is defined as in the preceding paragraph. Hence as in the previous computation

$$
\operatorname{deg} Z_{0}(f) \leq \operatorname{dim} \hat{\mathcal{O}}_{\mathbb{C}^{2}, 0} /\left\langle f_{x}, f_{y}\right\rangle+2 \operatorname{dim} \hat{\mathcal{O}}_{\mathbb{C}^{2}, 0} / I \leq 3 \mu(f)-2 \cdot \operatorname{mt}(f)+2 .
$$

\footnotetext{
${ }^{3}$ For the sake of notation we write $g$ instead of $\{g=0\}$ in these formulas.
} 


\section{AnAlytic And topological order \\ OF A ZERO-DIMENSIONAL SCHEME IN THE PLANE}

2.1. Definitions and notations. A zero-dimensional scheme $Z \subset \mathbb{P}^{2}$ can be characterized by the following numbers (orders):

$$
\begin{aligned}
& \operatorname{ord}_{0}(Z)=\min \left\{d \in \mathbb{Z}: H^{0}\left(\mathcal{J}_{Z / \mathbb{P}^{2}}(d)\right)>0\right\} \\
& \operatorname{ord}_{1}(Z)=\min \left\{d \in \mathbb{Z}: H^{1}\left(\mathcal{J}_{Z / \mathbb{P}^{2}}(d)\right)=0\right\}
\end{aligned}
$$

Put

$$
\operatorname{ord}_{0}^{t o p}(Z)=\max _{Z^{\prime} \in \operatorname{Def}(Z)} \operatorname{ord}_{0}\left(Z^{\prime}\right), \operatorname{ord}_{1}^{t o p}(Z)=\min _{Z^{\prime} \in \operatorname{Def}(Z)} \operatorname{ord}_{1}\left(Z^{\prime}\right)
$$

if $Z$ is a cluster scheme, and

$$
\operatorname{ord}_{0}^{a n}(Z)=\max _{Z^{\prime} \in \operatorname{Iso}(Z)} \operatorname{ord}_{0}\left(Z^{\prime}\right), \quad \operatorname{ord}_{1}^{a n}(Z)=\min _{Z^{\prime} \in \operatorname{Iso}(Z)} \operatorname{ord}_{i}\left(Z^{\prime}\right)
$$

if $Z$ is any scheme. Clearly, $\operatorname{ord}_{i}^{t o p}(Z), \operatorname{ord}_{i}^{a n}(Z)$ are $\operatorname{ord}_{i}\left(Z^{\prime}\right)$ for a generic element $Z^{\prime}$ of the corresponding family.

It was shown in 6], Lemmas 3.1, 4.1, 5.8, that

- for an irreducible zero-dimensional cluster scheme $Z$ defined by a curve germ having only nonsingular local branches,

$$
\operatorname{ord}_{1}^{t o p}(Z)<(1+\sqrt{2}) \sqrt{\operatorname{deg} Z}+\operatorname{mt} Z+1 .
$$

- for an arbitrary irreducible zero-dimensional cluster scheme $Z$,

$$
\operatorname{ord}_{1}^{t o p}(Z)<\frac{\sqrt{85}-3}{2} \sqrt{\operatorname{deg} Z}+\operatorname{mt} Z+\operatorname{mt}_{s} Z+1
$$

where $\mathrm{mt}_{s} Z$ is the sum of multiplicities of the singular branches of the germ $f(Z)$.

We shall estimate the analytic orders of any zero-dimensional scheme. As a byproduct we improve estimates (13), (14) for topological orders and extend them to reducible schemes.

Proposition 8. For any zero-dimensional scheme $Z \subset \mathbb{P}^{2}$,

$$
\operatorname{ord}_{0}^{a n}(Z) \geq \frac{\operatorname{deg} Z}{\sqrt{2 M_{2}(Z)}} .
$$

Remark 2. In view of (4), the bound (15) can be weakened up to the following, simpler inequality:

$$
\operatorname{ord}_{0}^{a n}(Z)>\frac{\sqrt{\operatorname{deg} Z}}{2} .
$$

Proof. Step 1. Consider, first, the case of an irreducible scheme $Z$ concentrated at a point $z \in \mathbb{P}^{2}$.

Let $Y$ be a generic element of $\operatorname{Iso}(Z)$, concentrated at $z$, and $d=\operatorname{ord}_{0}(Y)$. Take a generic curve $C \in\left|\mathcal{J}_{Y / \mathbb{P}^{2}}(d)\right|$, and suppose that $C=C_{1}^{l_{1}} \ldots C_{r}^{l_{r}}$, where $C_{1}, \ldots, C_{r}$ are distinct reduced irreducible curves of degrees $d_{1}, \ldots, d_{r}$, respectively, so that $d=l_{1} d_{1}+\ldots+l_{r} d_{r}$.

If $C$ contains only point $z$ of $T\left(Y_{c l}\right)$, then $C$ transversally intersects a generic element $f \in I(Y)$. Since $d \geq \operatorname{mt}\left(Y_{c l}, z\right)$, we have

$$
d \geq \sqrt{d \cdot \operatorname{mt}\left(Y_{c l}, z\right)}=\sqrt{(C \cdot f)_{z}} \geq \sqrt{\operatorname{deg} Y}=\sqrt{\operatorname{deg} Z} .
$$


Now, without loss of generality, we can assume that the tree $T\left(Y_{c l}\right)$ contains at least two points, the curves $C_{i}, 1 \leq i \leq s<r$, contain only point $z$ of $T\left(Y_{c l}\right)$, and any curve $C_{i}, i>s$, contains at least two points of $T\left(Y_{c l}\right)$.

Fix $s<i \leq r$. Put $T_{i}=T\left(C_{i}\right) \cap T\left(Y_{c l}\right)$. Choose local coordinates $x, y$ in a neighborhood of $z=(0,0)$ so that the axes are transverse to $C_{i}$ and to a generic $f \in I(Y)$. Introduce the sequence

$$
\psi_{m} \in \operatorname{Aut}\left(\hat{\mathcal{O}}_{\mathbb{P}^{2}, z}\right), \quad \psi_{m}(x, y)=\left(x, y+\varepsilon x^{m}\right), \quad m \geq 1, \quad \varepsilon=\mathrm{const} \neq 0,
$$

and consider the schemes $\psi_{m}(Y)$. The ascending sequence of trees $T\left(Y_{c l}\right) \cap$ $T\left(\psi_{m}\left(Y_{c l}\right)\right)$ stabilizes for a sufficiently large $m$. Then there exists

$$
k=\max \left\{m \geq 1: T_{i} \not \subset T\left(\psi_{m}\left(Y_{c l}\right)\right)\right\} .
$$

\section{Lemma 9.}

$$
\sum_{q \in T_{i} \backslash T\left(\psi_{k}\left(Y_{c l}\right)\right)}\left(\operatorname{mt}\left(C_{i}, q\right)\right)^{2} \leq\left(\operatorname{mt}\left(C_{i}, z\right)\right)^{2} .
$$

Proof of Lemma 9. Denote by $T_{m}\left(C_{i}\right), m \geq 1$, the tree of common infinitely near points of the curves $C_{i}$ and $\psi_{m}\left(C_{i}\right)$ at $z$. Then

$$
\begin{gathered}
T_{i} \backslash T\left(\psi_{k}\left(Y_{c l}\right)\right) \subset T_{k+1}\left(C_{i}\right) \backslash T_{k}\left(C_{i}\right) \\
\sum_{q \in T_{i} \backslash T\left(\psi_{k}\left(Y_{c l}\right)\right)}\left(\operatorname{mt}\left(C_{i}, q\right)\right)^{2} \leq \sum_{q \in T_{k+1}\left(C_{i}\right) \backslash T_{k}\left(C_{i}\right)}\left(\operatorname{mt}\left(C_{i}, q\right)\right)^{2} \\
=\left(C_{i} \cdot \psi_{k+1} C_{i}\right)_{z}-\left(C_{i} \cdot \psi_{k} C_{i}\right)_{z} .
\end{gathered}
$$

To estimate the latter expression, we use the Puiseux decomposition

$$
C_{i}(x, y)=(1+O(x, y)) \prod_{s=1}^{n}\left(y-\xi_{s}(x)\right), \quad n=\operatorname{mt}\left(C_{i}, z\right),
$$

where $\xi_{s}(x), s=1, \ldots, n$, are fractional power series. Then

$$
\begin{aligned}
\left(C_{i} \cdot \psi_{k}\left(C_{i}\right)\right)_{z} & =\sum_{1 \leq r, s \leq n} \operatorname{ord}\left(\xi_{r}(x)-\xi_{s}(x)-\varepsilon x^{k}\right), \\
\left(C_{i} \cdot \psi_{k+1}\left(C_{i}\right)\right)_{z} & =\sum_{1 \leq r, s \leq n} \operatorname{ord}\left(\xi_{r}(x)-\xi_{s}(x)-\varepsilon x^{k+1}\right),
\end{aligned}
$$

and (18) follows, because of an obvious inequality

$$
\operatorname{ord}\left(\xi_{r}(x)-\xi_{s}(x)-\varepsilon x^{k+1}\right) \leq \operatorname{ord}\left(\xi_{r}(x)-\xi_{s}(x)-\varepsilon x^{k}\right)+1,
$$

where $\operatorname{ord}(*)$ means the minimal power of $x$ occurring in the series $*$.

In view of the generality of $Y \in \operatorname{Iso}(Z)$ and $\varepsilon \neq 0$, the scheme $Y^{\prime}=\psi_{k}(Y)$ is also generic in $\operatorname{Iso}_{z}(Z)$. Hence $\operatorname{ord}_{0}\left(Y^{\prime}\right)=\operatorname{ord}_{0}(Y)=d$, and $Y^{\prime}$ is contained in a curve $C^{\prime}$ of degree $d$ which splits as $C^{\prime}=\left(C_{1}^{\prime}\right)^{l_{1}} \ldots\left(C_{r}^{\prime}\right)^{l_{r}}$, where $\operatorname{deg} C_{i}^{\prime}=\operatorname{deg} C_{i}=d_{i}$, and the curve $C_{i}^{\prime}$ is different from $C_{i}$, since $\psi_{k}$ moves the points $q \in T_{i} \backslash T\left(Y_{c l}^{\prime}\right) \neq \emptyset$. Then

$$
d_{i}^{2} \geq \sum_{q \in T\left(Y_{c l}\right) \cap T\left(Y_{c l}^{\prime}\right)}\left(\operatorname{mt}\left(C_{i}, q\right)\right)^{2},
$$

which by (18) implies

$$
d_{i}^{2} \geq \frac{1}{2} \sum_{q \in T\left(Y_{c l}\right)}\left(\operatorname{mt}\left(C_{i}, q\right)\right)^{2}
$$


Note that (20) holds for $i=1, \ldots, s$ as well.

Take a generic element $f \in I(Y)$. Then $Y$ is contained in the scheme-theoretic intersection of $f$ and $C$ at the point $z$. Hence

$$
\begin{gathered}
\operatorname{deg} Y=\operatorname{deg} Z \leq(f \cdot C)_{z}=\sum_{q \in T\left(Y_{c l}\right)} \operatorname{mt}\left(Y_{c l}, q\right) \cdot \operatorname{mt}(C, q) \\
\leq \sqrt{\sum_{q \in T\left(Y_{c l}\right)}\left(\operatorname{mt}\left(Y_{c l}, q\right)\right)^{2}} \sqrt{\sum_{q \in T\left(Y_{c l}\right)}(\operatorname{mt}(C, q))^{2}}=\sqrt{M_{2}(Z)} \sqrt{\sum_{q \in T\left(Y_{c l}\right)}(\operatorname{mt}(C, q))^{2}}
\end{gathered}
$$

Here

$$
\sum_{q \in T\left(Y_{c l}\right)}(\operatorname{mt}(C, q))^{2}=\sum_{q \in T\left(Y_{c l}\right)}\left(\sum_{i=1}^{r} l_{i} \cdot \operatorname{mt}\left(C_{i}, q\right)\right)^{2} \stackrel{(20)}{\leq} 2\left(\sum_{i=1}^{r} l_{i} d_{i}\right)^{2}=2 d^{2},
$$

and (15) follows.

Step 2. Let $Z$ consist of components $Z_{1}, \ldots, Z_{p}$ concentrated at points $z_{1}, \ldots, z_{p} \in$ $\mathbb{P}^{2}$, respectively, $p \geq 2$. Consider a generic scheme $Y \in \operatorname{Iso}(Z)$, concentrated at a generic $p$-tuple $w_{1}, \ldots, w_{p} \in \mathbb{P}^{2}$, and a generic curve $C \in\left|\mathcal{J}_{Y / \mathbb{P}^{2}}(d)\right|, d=\operatorname{ord}_{0}(Y)=$ $\operatorname{ord}_{0}^{a n}(Z)$. Assume that $C=C_{1}^{l_{1}} \ldots C_{r}^{l_{r}}$, where $C_{1}, \ldots, C_{r}$ are reduced irreducible.

If a component $C_{i}, 1 \leq i \leq r$, passes through only one of the points $w_{1}, \ldots, w_{p}$, then (20) holds due to the argument in Step 1 of the proof.

Let $C_{i}$ pass through points $w_{1}, \ldots, w_{s}, s \geq 2$, and be transverse to generic elements $f_{j} \in I\left(Y_{w_{j}}\right), j=1, \ldots, s$. Assuming that $0<\operatorname{mt}\left(C_{i}, w_{1}\right) \leq \ldots \leq \operatorname{mt}\left(C_{i}, w_{k}\right)$, we move the point $w_{1}$ to $w_{1}^{\prime} \notin C_{i}$, keeping $w_{2}, \ldots, w_{p}$ fixed. The correspondingly deformed scheme $Y^{\prime}$ is also generic in $\operatorname{Iso}(Z)$, and hence there exists a curve $C^{\prime} \in\left|\mathcal{J}_{Y^{\prime} / \mathbb{P}^{2}}(d)\right|$, splitting as $C^{\prime}=\left(C_{1}^{\prime}\right)^{l_{1}} \ldots\left(C_{r}^{\prime}\right)^{l_{r}}$ with $\operatorname{deg} C_{j}^{\prime}=d_{j}, j=1, \ldots, r, C_{i}^{\prime}$ close to $C_{i}$ having multiplicities

$$
\operatorname{mt}\left(C_{i}^{\prime}, w_{1}^{\prime}\right)=\operatorname{mt}\left(C_{i}, w_{1}\right), \quad \operatorname{mt}\left(C_{i}^{\prime}, w_{j}\right)=\operatorname{mt}\left(C_{i}, w_{j}\right), j=2, \ldots, k .
$$

Then by [8], Theorem 2(1), or [24], Lemma 3,

$$
\begin{aligned}
d_{i}^{2} & \geq\left(\operatorname{mt}\left(C_{i}, w_{1}\right)\right)^{2}-\operatorname{mt}\left(C_{i}, w_{1}\right)+\sum_{j=2}^{k}\left(\operatorname{mt}\left(C_{i}, w_{j}\right)\right)^{2} \\
& \geq \frac{1}{2} \sum_{j=1}^{k}\left(\operatorname{mt}\left(C_{i}, w_{j}\right)\right)^{2}=\frac{1}{2} \sum_{q \in T\left(Y_{c l}\right)}\left(\operatorname{mt}\left(C_{i}, q\right)\right)^{2} .
\end{aligned}
$$

Let $C_{i}$ pass through $w_{1}, \ldots, w_{s}, s \geq 2$, and contain at least two points of $T\left(Y_{w_{1}, c l}\right)$. Then we apply transformations (16) to $Y_{w_{1}}$, where $w_{1}=(0,0)$, keeping $Y_{w_{j}}, j=$ $2, \ldots, p$, unchanged. The reasoning, similar to that in Step 1 of the proof, shows that a suitable $\psi_{m}$ moves the tree $T\left(Y_{w_{1}, c l}\right) \cap T\left(C_{i}\right)$, turning $C_{i}$ into a curve $C_{i}^{\prime}$ of the same degree $d_{i}$ with

$$
\left(C_{i} \cdot C_{i}^{\prime}\right)_{w_{1}} \geq \frac{1}{2} \sum_{q \in T\left(Y_{w_{1}, c l}\right) \cap C_{i}}\left(\operatorname{mt}\left(C_{i}, q\right)\right)^{2},
$$


which immediately implies

$$
\begin{gathered}
d_{i}^{2} \geq \frac{1}{2} \sum_{q \in T\left(Y_{w_{1}, c l}\right) \cap C_{i}}\left(\operatorname{mt}\left(C_{i}, q\right)\right)^{2}+\sum_{j=2}^{k} \sum_{q \in T\left(Y_{w_{j}, c l}\right) \cap C_{i}}\left(\operatorname{mt}\left(C_{i}, q\right)\right)^{2} \\
\geq \frac{1}{2} \sum_{q \in T\left(Y_{c l}\right) \cap C_{i}}\left(\operatorname{mt}\left(C_{i}, q\right)\right)^{2} .
\end{gathered}
$$

Finally, (15) follows from (20), (21), (22), as was done in Step 1 of the proof.

Proposition 10. For any singular zero-dimensional scheme $Z \subset \mathbb{P}^{2}$,

$$
\operatorname{ord}_{1}^{a n}(Z) \leq \sqrt{\frac{3}{2} M_{2}(Z)}+\frac{\operatorname{deg} Z}{\sqrt{3 M_{2}(Z) / 2}}-2 .
$$

For any nonsingular zero-dimensional cluster scheme $Z \subset \mathbb{P}^{2}$,

$$
\operatorname{ord}_{1}^{a n}(Z)=-\left[\frac{3-\sqrt{1+8 \operatorname{deg} Z}}{2}\right] \text {. }
$$

Remark 3. Due to (4), inequality (23) implies a weaker relation

$$
\operatorname{ord}_{1}^{a n}(Z)<\frac{4}{\sqrt{3}} \sqrt{\operatorname{deg} Z}-2,
$$

which in turn is stronger than both (13) and (14).

Proof. Step 1. Let $Z$ be nonsingular (and hence a cluster scheme). Relation (24) means that

$$
d=\operatorname{ord}_{1}^{a n}(Z)=\min \left\{n: h^{0}\left(\hat{\mathcal{O}}_{\mathbb{P}^{2}}(n)\right)=\frac{(n+1)(n+2)}{2} \geq \operatorname{deg} Z\right\} .
$$

In other words, the $\operatorname{deg} Z$ conditions imposed on curves $C \in\left|\mathcal{J}_{Y / \mathbb{P}^{2}}(d)\right|$ by a generic element $Y \in \operatorname{Def}(Z)=\operatorname{Iso}(Z)$ are independent. Take a natural sequence of schemes $\emptyset \subsetneq Z_{1} \subsetneq \ldots \subsetneq Z_{s}=Z, s=\operatorname{deg} Z$, where the linear system $\left|\mathcal{J}_{Z_{i+1} / \mathbb{P}^{2}}(d)\right|$ is obtained from $\left|\mathcal{J}_{Z_{i} / \mathbb{P}^{2}}(d)\right|$ by imposing one condition, either a passage through a point $\notin Z_{i}$, or an extended by 1 tangency order with a fixed nonsingular curve germ. Then one can inductively show that generic members of these linear systems are nonsingular, and a generic choice of the new condition reduces the dimension each time by 1 .

Step 2. Take a generic scheme $Y \in \operatorname{Iso}(Z)$. Consider the Castelnuovo function $\mathcal{C}_{Y}(n)=h^{1}\left(\mathcal{J}_{Y / \mathbb{P}^{2}}(n-1)\right)-h^{1}\left(\mathcal{J}_{Y / \mathbb{P}^{2}}(n)\right), n \geq 0$. One can find a detailed description of the Castelnuovo function and its graph in [3, 7. In particular (see Figure 1),

$$
\begin{gathered}
\mathcal{C}_{Y}(n)=n+1,0 \leq n<\operatorname{ord}_{0}(Y)=\operatorname{ord}_{0}^{a n}(Z), \\
\mathcal{C}_{Y}(n) \leq \mathcal{C}_{Y}(n-1), n \geq \operatorname{ord}_{0}(Y), \\
\mathcal{C}_{Y}(n)=0, n>\operatorname{ord}_{1}(Y)=\operatorname{ord}_{1}^{a n}(Z), \\
\sum_{n \geq 0} \mathcal{C}_{Y}(n)=\operatorname{deg} Z=\operatorname{deg} Y .
\end{gathered}
$$

If there is no $n \geq \operatorname{ord}_{0}(Y)$ such that $\operatorname{ord}_{0}(Y)>\mathcal{C}_{Y}(n)=\mathcal{C}_{Y}(n-1)>0$, then we put $d=\operatorname{ord}_{0}(Y)$. If there exists $0<d<\operatorname{ord}_{0}(Y)$ such that $\mathcal{C}_{Y}(n)=\mathcal{C}_{Y}(n-1)=d$ for some $n \geq \operatorname{ord}_{0}(Y)$ (so-called "long stair"), then we assume that $d$ is minimal with this property (see Figure 1). By [3], Claims 2.2 and 2.3, (see also [7]), there exists a curve $C$ of degree $d$ such that (see Figure 1)

$$
\mathcal{C}_{C \cap Y}(n)=\min \left\{d, \mathcal{C}_{Y}(n)\right\} .
$$




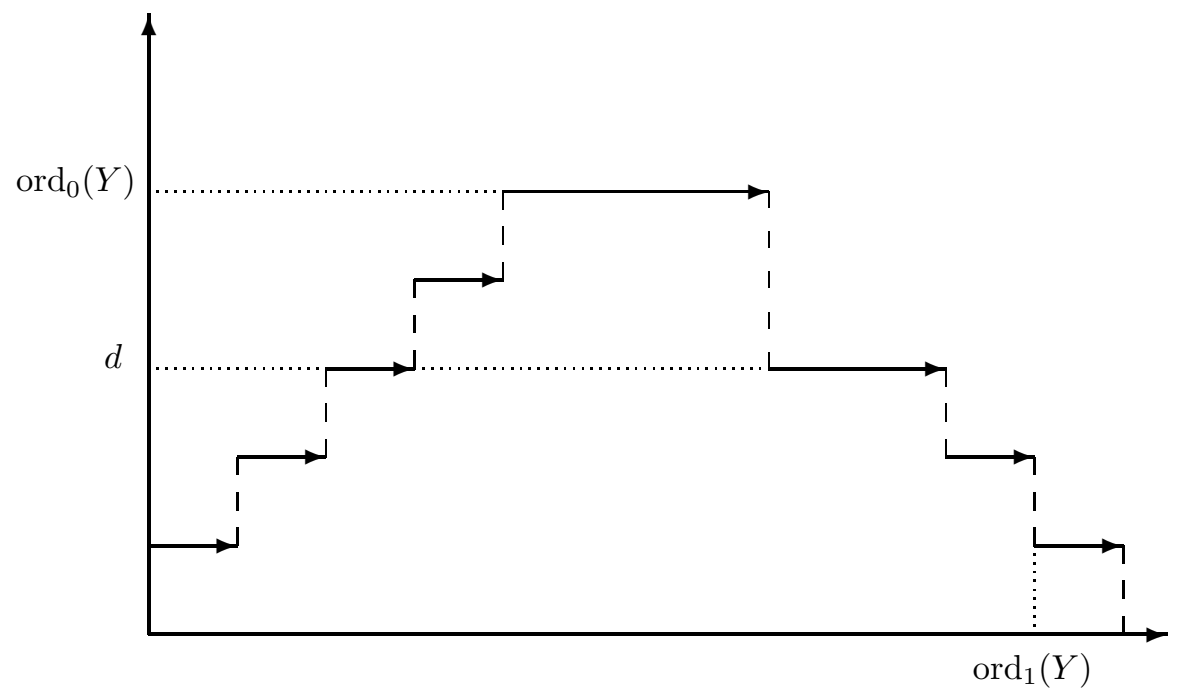

FiguRE 1. Castelnuovo function

At this moment we assume that $d>\sqrt{\operatorname{deg}(Y \cap C)}$. Taking into account the choice of $d$ and property (29), and looking at Figure 1] we derive that

$$
2 d>\operatorname{ord}_{1}(Y)+1 \quad \Longrightarrow \quad d \geq \frac{\operatorname{ord}_{1}(Y)}{2}+1
$$

and consequently

$$
\operatorname{deg}(Y \cap C) \geq \frac{d(d+1)}{2}+\frac{\left(\operatorname{ord}_{1}(Y)+1-d\right)\left(\operatorname{ord}_{1}(Y)+2-d\right)}{2} \geq \frac{\left(\operatorname{ord}_{1}(Y)+2\right)^{2}}{4} .
$$

Hence, in view of $M_{2}(Y) \geq \operatorname{deg} Y \geq \operatorname{deg}(Y \cap C)$,

$$
\operatorname{ord}_{1}(Y) \leq 2 \sqrt{\operatorname{deg}(Y \cap C)}-2<\sqrt{\frac{3}{2} M_{2}(Y)}+\frac{\operatorname{deg} Y}{\sqrt{3 M_{2}(Y) / 2}}-2 .
$$

Step 3. From now on we assume that $Z$ is singular, and, in the notation of Step 2 ,

$$
d \leq \sqrt{\operatorname{deg}(Y \cap C)} .
$$

For the reader's convenience we start by proving a bound weaker than (23),

$$
\operatorname{ord}_{1}^{a n}(Z) \leq \sqrt{2 M_{2}(Z)}+\frac{\operatorname{deg} Z}{\sqrt{2 M_{2}(Z)}}-2
$$

It will illustrate the main idea of the proof, which is based on the argument used in the proof of Proposition 8. Further refinement up to inequality (23) is of a technical nature and consists of exploring particular steps in the proof of (32).

Remark 4. The use of inequality (32) instead of (23) leads, in fact, to similar estimates of orders of singular points with a different constant factor. 
The choice of $d$ and properties (26)-(29) of the Castelnuovo function immediately imply that

$$
\begin{aligned}
& \left.\operatorname{ord}_{1}(Y)+1\right) d \leq \operatorname{deg}(Y \cap C)+d(d-1) \\
& \Longrightarrow \quad \operatorname{ord}_{1}^{a n}(Z) \leq \frac{\operatorname{deg}(Y \cap C)}{d}+d-2 .
\end{aligned}
$$

We shall estimate $d=\operatorname{deg} C$ from below using the argument in the proof of Proposition 8 . The generality of $Y$ in $\operatorname{Iso}(Z)$ guarantees the fixed shape of $\mathcal{C}_{Y}$ when varying $Y$ in an open dense subset of $\operatorname{Iso}(Z)$, as well as the existence of a continuous family of curves $C$ of degree $d$, satisfying (30) and having the same collection of degrees of irreducible components. Let $C=C_{1}^{l_{1}} \ldots C_{r}^{l_{r}}$, where $C_{1}, \ldots, C_{r}$ are reduced irreducible, $\operatorname{deg} C_{i}=d_{i}, i=1, \ldots, r$. Then, reasoning as in the proof of Proposition 8 we obtain

$$
d_{i}^{2} \geq \frac{1}{2} \sum_{q \in T\left(Y_{c l}\right)}\left(\operatorname{mt}\left(C_{i}, q\right)\right)^{2}, \quad i=1, \ldots, r
$$

Taking generic elements $f_{j} \in I\left(Y_{w_{j}}\right), Y=Y_{w_{1}} \cup \ldots \cup Y_{w_{p}}, w_{1}, \ldots, w_{p} \in \mathbb{P}^{2}$, one obtains

$$
\begin{aligned}
\operatorname{deg}(C \cap Y) \leq & \sum_{j=1}^{p}\left(f_{j} \cdot C\right)_{w_{j}} \leq \sqrt{\sum_{q \in T\left(Y_{c l}\right)}(\operatorname{mt}(Y, q))^{2}} \sqrt{\sum_{q \in T\left(Y_{c l}\right)}(\operatorname{mt}(C, q))^{2}} \\
& \leq \sqrt{2 M_{2}(Y)} \cdot d \Longrightarrow d \geq \frac{\operatorname{deg}(C \cap Y)}{\sqrt{2 M_{2}(Y)}}
\end{aligned}
$$

In view of this bound and (31), inequality (33) implies (32).

Step 4. To refine inequality (32) we need a strengthened form of Lemma 9

Lemma 11. In the notation of Step 3 , either $C_{i}$ is a straight line containing two points of $T\left(Y_{c l}\right)$, or

$$
d_{i}^{2} \geq \frac{2}{3} \sum_{q \in T\left(Y_{c l}\right)}\left(\operatorname{mt}\left(C_{i}, q\right)\right)^{2} .
$$

The proof is found in section 2.2 below.

We shall now prove (23) for schemes $Z$ such that, for any two points $q_{1}, q_{2} \in$ $T\left(Z_{c l}\right)$,

$$
\operatorname{mt}\left(Z_{c l}, q_{1}\right)+\operatorname{mt}\left(Z_{c l}, q_{2}\right) \leq \sqrt{\frac{3}{2} M_{2}(Z)}
$$

In the notation of Step 2, let $C=C^{\prime} C^{\prime \prime}, C^{\prime}=C_{1}^{l_{1}} \ldots C_{s}^{l_{s}}, C^{\prime \prime}=C_{s+1}^{l_{s+1}} \ldots C_{r}^{l_{r}}$, where $0 \leq s \leq r$; any $C_{i}, i=1, \ldots, s$, is a straight line containing exactly two points of $T\left(Y_{c l}\right)$, and $C_{s+1}, \ldots, C_{r}$ are the other irreducible components of $C$.

Taking generic elements $f_{j} \in I\left(Y_{w_{j}}\right), Y=Y_{w_{1}} \cup \ldots \cup Y_{w_{p}}, w_{1}, \ldots, w_{p} \in \mathbb{P}^{2}$, one obtains

$$
\operatorname{deg}\left(Y \cap C^{\prime \prime}\right) \leq \sum_{j=1}^{p}\left(f_{j} \cdot C^{\prime \prime}\right)_{w_{j}} \leq \sqrt{\sum_{q \in T\left(Y_{c l}\right)}\left(\operatorname{mt}\left(Y_{c l}, q\right)\right)^{2}} \sqrt{\sum_{q \in T\left(Y_{c l}\right)}\left(\operatorname{mt}\left(C^{\prime \prime}, q\right)\right)^{2}} .
$$


By Lemma 11

$$
\begin{gathered}
\sqrt{\sum_{q \in T\left(Y_{c l}\right)}\left(\operatorname{mt}\left(C^{\prime \prime}, q\right)\right)^{2}}=\sum_{q \in T\left(Y_{c l}\right)}\left(\sum_{i=s+1}^{r} l_{i} \cdot \operatorname{mt}\left(C_{i}, q\right)\right)^{2} \\
\leq \sum_{i=s+1}^{r} l_{i} \sqrt{\sum_{q \in T_{i}}\left(\operatorname{mt}\left(C_{i}, q\right)\right)^{2}} \leq \sqrt{\frac{3}{2}} \sum_{i=s+1}^{r} l_{i} d_{i} .
\end{gathered}
$$

Hence

$$
\operatorname{deg}\left(Y \cap C^{\prime \prime}\right) \leq \sqrt{\frac{3}{2} M_{2}(Z)} \sum_{i=s+1}^{r} l_{i} d_{i} .
$$

On the other hand, by (35),

$$
\begin{gathered}
\operatorname{deg}\left(Y \cap C^{\prime}\right) \leq \sum_{j=1}^{p}\left(f_{j} \cdot C^{\prime}\right)_{w_{j}}=\sum_{i=1}^{s} l_{i}\left(\sum_{j=1}^{p}\left(f_{j} \cdot C_{i}\right)_{w_{j}}\right) \\
\leq \sqrt{\frac{3}{2} M_{2}(Z)} \sum_{i=1}^{s} l_{i} .
\end{gathered}
$$

So, it follows that

$$
\begin{gathered}
\operatorname{deg}(Y \cap C) \leq \operatorname{deg}\left(Y \cap C^{\prime}\right)+\operatorname{deg}\left(Y \cap C^{\prime \prime}\right) \leq d \sqrt{\frac{3}{2} M_{2}(Z)} \\
\Longrightarrow \quad d \geq \frac{\operatorname{deg}(Y \cap C)}{\sqrt{3 M_{2}(Z) / 2}},
\end{gathered}
$$

which implies (23) by virtue of (33) and (31).

Step 5. We shall complete the proof of Proposition 10 by induction on $\operatorname{deg} Z$. The case of nonsingular $Z$ of degree $\neq 2$ is the base of induction since (24) implies (23) except for $\operatorname{deg} Z=2$.

Assume that $Z$ is singular. By the result of Step 4, one has to consider only the case that $T(Z)$ has at least two vertices, and there exist $q_{1}, q_{2} \in T(Z)$ such that

$$
\operatorname{mt}\left(Z_{c l}, q_{1}\right)+\operatorname{mt}\left(Z_{c l}, q_{2}\right)>\sqrt{\frac{3}{2} M_{2}(Z)} .
$$

This means that, for a generic $Y \in \operatorname{Iso}(Z)$, there exists a straight line $L$ containing two points of $T(Y)$ and satisfying

$$
\operatorname{deg}(Y \cap L)>\sqrt{\frac{3}{2} M_{2}(Z)}=\sqrt{\frac{3}{2} M_{2}(Y)} .
$$

In other words, $L$ contains two vertices $q_{1}, q_{2}$ of $T\left(Y_{c l}\right)$ with multiplicities $m_{i}=$ $\operatorname{mt}\left(Y_{c l}, q_{i}\right), i=1,2, m_{1} \geq 2$, and

$$
\operatorname{deg}(Y \cap L) \geq m_{1}+m_{2}>\sqrt{\frac{3}{2} \sum_{q \in T\left(Y_{c l}\right)}\left(\operatorname{mt}\left(Y_{c l}, q\right)\right)^{2}} .
$$

Put

$$
d=\left[\sqrt{\frac{3}{2} M_{2}(Z)}+\frac{\operatorname{deg} Z}{\sqrt{3 M_{2}(Z) / 2}}-2\right] .
$$


First, notice that

$$
d \geq m_{1}+m_{2}-1
$$

Indeed,

$$
\begin{gathered}
\sqrt{\frac{3}{2} M_{2}(Z)}+\frac{\operatorname{deg} Z}{\sqrt{3 M_{2}(Z) / 2}}-2 \geq \sqrt{\frac{3}{2} M_{2}(Z)}+\frac{\left(m_{1}\left(m_{1}+1\right)+m_{2}\left(m_{2}+1\right)\right) / 2}{\sqrt{3 M_{2}(Z) / 2}}-2 \\
\geq \sqrt{\frac{3}{2}\left(m_{1}^{2}+m_{2}^{2}\right)}+\frac{m_{1}\left(m_{1}+1\right)+m_{2}\left(m_{2}+1\right)}{\sqrt{6\left(m_{1}^{2}+m_{2}^{2}\right)}}-2 \geq m_{1}+m_{2}-1
\end{gathered}
$$

where the latter inequality holds, because substituting $m_{1}$ and $m_{2}$ for $m=$ $\left(m_{1}+m_{2}\right) / 2$, we diminish the left-hand side and obtain an inequality

$$
\frac{4}{\sqrt{3}} m+\frac{1}{\sqrt{3}}-2 \geq 2 m-1
$$

which holds true for $m \geq 3 / 2$.

The exact sequence of sheaves

$$
0 \rightarrow \mathcal{J}_{Y: L / \mathbb{P}^{2}}(d-1) \rightarrow \mathcal{J}_{Y / \mathbb{P}^{2}}(d) \rightarrow \mathcal{J}_{Y \cap L / L}(d) \rightarrow 0
$$

induces the exact cohomology sequence

$$
H^{1}\left(\mathcal{J}_{Y: L / \mathbb{P}^{2}}(d-1)\right) \rightarrow H^{1}\left(\mathcal{J}_{Y / \mathbb{P}^{2}}(d)\right) \rightarrow H^{1}\left(\mathcal{J}_{Y \cap L / L}(d)\right)
$$

where the last term vanishes due to (38). Hence $H^{1}\left(\mathcal{J}_{Y / \mathbb{P}^{2}}(d)\right)=0$, provided $H^{1}\left(\mathcal{J}_{Y: L / \mathbb{P}^{2}}(d-1)\right)=0$.

If $\operatorname{deg}(Y: L) \neq 2$, by the induction assumption, inequality (36), and the formula $\operatorname{deg}(Y: L)=\operatorname{deg} Y-m_{1}-m_{2}$,

$$
\begin{gathered}
\operatorname{ord}_{1}(Y: L) \leq \sqrt{\frac{3}{2} M_{2}(Y: L)}+\frac{\operatorname{deg} Y: L}{\sqrt{3 M_{2}(Y: L) / 2}}-2 \\
\leq \sqrt{\frac{3}{2} M_{2}(Y)}+\frac{\operatorname{deg} Y-m_{1}-m_{2}}{\sqrt{3 M_{2}(Y) / 2}}-2<\sqrt{\frac{3}{2} M_{2}(Y)}+\frac{\operatorname{deg} Y}{\sqrt{3 M_{2}(Y) / 2}}-3 \\
\Longrightarrow \operatorname{ord}_{1}(Y: L) \leq d-1,
\end{gathered}
$$

and we are done.

If $\operatorname{deg}(Y: L)=2$, then, clearly, $H^{1}\left(\mathcal{J}_{Y: L / \mathbb{P}^{2}}(d-1)\right)=0$ holds, provided $d \geq 2$, which follows from (37), since $m_{1} \geq 2, m_{2} \geq 1$, and consequently $\operatorname{deg} Z \geq 4$.

2.2. Proof of Lemma 11. Without loss of generality we assume that $T\left(Y_{c l}\right)$ has at least two vertices, $C_{i}$ contains at least two points of $T\left(Y_{c l}\right)$, and is not a straight line, containing exactly two points of $T\left(Y_{c l}\right)$.

Let $C_{i}$ contain exactly two points, say $q_{1}, q_{2}$ of $T\left(Y_{c l}\right)$, and is singular at $q_{1}, q_{2}$. Then $d_{i} \geq 2$, and (34) turns into the inequality $d_{i}^{2} \geq 4 \geq 2 / 3(1+1)$.

Let $C_{i}$ contain exactly two points of $T\left(Y_{c l}\right)$. If one of these points $q$ is infinitely near to the other point $z$, and $C_{i}$ is singular at $z$, i.e., $\operatorname{mt}\left(C_{i}, z\right) \geq 2$, then, intersecting the curves $C_{i}$ and $\psi_{1}\left(C_{i}\right)$ (see the notation in the proof of Lemma 9), we obtain by 8], Theorem 2(1), or [24], Lemma 3 ,

$$
d_{i}^{2} \geq\left(\operatorname{mt}\left(C_{i}, z\right)\right)^{2}+\left(\operatorname{mt}\left(C_{i}, q\right)\right)^{2}-\operatorname{mt}\left(C_{i}, q\right) \geq \frac{2}{3}\left(\left(\operatorname{mt}\left(C_{i}, z\right)\right)^{2}+\left(\operatorname{mt}\left(C_{i}, q\right)\right)^{2}\right),
$$

where the latter inequality follows from

$$
\left(\operatorname{mt}\left(C_{i}, z\right)\right)^{2}+\left(\operatorname{mt}\left(C_{i}, q\right)\right)^{2}-3 \cdot \operatorname{mt}\left(C_{i}, q\right) \geq 4+\left(\operatorname{mt}\left(C_{i}, q\right)\right)^{2}-3 \cdot \operatorname{mt}\left(C_{i}, q\right)>0 .
$$


If $C_{i} \cap T\left(Y_{c l}\right)$ consists of points $z_{1} \neq z_{2} \in \mathbb{P}^{2}$, and $C_{i}$ is singular at $z_{1}$, i.e., $\operatorname{mt}\left(C_{i}, z_{1}\right) \geq 2$, then, moving the point $z_{2}$ and respectively the curve $C_{i}$, we similarly obtain

$d_{i}^{2} \geq\left(\operatorname{mt}\left(C_{i}, z_{1}\right)\right)^{2}+\left(\operatorname{mt}\left(C_{i}, z_{2}\right)\right)^{2}-\operatorname{mt}\left(C_{i}, z_{2}\right) \geq \frac{2}{3}\left(\left(\operatorname{mt}\left(C_{i}, z_{1}\right)\right)^{2}+\left(\operatorname{mt}\left(C_{i}, z_{2}\right)\right)^{2}\right)$.

The previous argument in general leads to (34), when $C_{i} \cap Y$ is reducible. So, we assume that $C_{i} \cap Y$ is irreducible, and $C_{i}$ contains at least three points of $T\left(Y_{c l}\right)$.

Let $k \geq 2$ in (17). We shall refine the statement of Lemma 9 up to (see the notation of Lemma 9)

$$
\sum_{q \in T_{i} \backslash T\left(\psi_{k}(Y)\right)}\left(\operatorname{mt}\left(C_{i}, q\right)\right)^{2} \leq \frac{1}{k+1} \sum_{q \in T_{i}}\left(\operatorname{mt}\left(C_{i}, q\right)\right)^{2} .
$$

Indeed, it would immediately follow from

$$
\sum_{q \in T_{i} \cap T\left(\psi_{m+1}(Y)\right) \backslash T\left(\psi_{m}(Y)\right)}\left(\operatorname{mt}\left(C_{i}, q\right)\right)^{2} \leq \sum_{q \in T_{i} \cap T\left(\psi_{m}(Y)\right) \backslash T\left(\psi_{m-1}(Y)\right)}\left(\operatorname{mt}\left(C_{i}, q\right)\right)^{2}
$$

for all $m \geq 1$. The vertices of $T\left(C_{i}\right), T(Y)$ are encoded by finite segments of the Puiseux expansion in a neighborhood of $z=(0,0)$

$$
\begin{array}{cc}
C_{i}(x, y) & =(1+O(x, y)) \prod_{s=1}^{n}\left(y-\xi_{s}(x)\right), \quad n=\operatorname{mt}\left(C_{i}, z\right), \\
f(Y)(x, y) & =(1+O(x, y)) \prod_{s=1}^{m_{0}}\left(y-\eta_{s}(x)\right), \quad m_{0}=\operatorname{mt}(Y, z),
\end{array}
$$

where $\xi_{s}(x), \eta(x)$ are fractional power series. For any $s=1, \ldots, n$, there exists $p(s)$ such that

$$
\operatorname{ord}\left(\xi_{s}(x)-\eta_{p(s)}(x)\right)=\max _{j} \operatorname{ord}\left(\xi_{s}(x)-\eta_{j}(x)\right)
$$

Then

$$
\begin{gathered}
\sum_{q \in T_{i}}\left(\operatorname{mt}\left(C_{i}, q\right)\right)^{2}=\sum_{s=1}^{n} \sum_{t=1}^{n} \min \left\{\operatorname{ord}\left(\xi_{t}(x)-\xi_{s}(x)\right), \operatorname{ord}\left(\xi_{t}(x)-\eta_{p(s)}\right)\right\} \\
=\sum_{s=1}^{n} \sum_{t=1}^{n} \operatorname{ord}\left(\xi_{t}(x)-\frac{\xi_{s}(x)+\varepsilon_{1} \eta_{p(s)}(x)}{1+\varepsilon_{1}}\right)
\end{gathered}
$$

for a generic number $\varepsilon_{1}$. Similarly,

$$
\sum_{q \in T_{i} \cap T\left(\psi_{m} Y\right)}\left(\operatorname{mt}\left(C_{i}, q\right)\right)^{2}=\sum_{s=1}^{n} \sum_{t=1}^{n} \operatorname{ord}\left(\xi_{t}(x)-\frac{\xi_{s}(x)+\varepsilon_{1} \eta_{p(s)}(x)}{1+\varepsilon_{1}}-\varepsilon x^{m}\right),
$$


for generic numbers $\varepsilon_{1}, \varepsilon$. In view of the last relation, the following series of simple inequalities yields (40) and thereby (39):

$$
\begin{aligned}
& \operatorname{ord}\left(\xi_{t}(x)-\frac{\xi_{s}(x)+\varepsilon_{1} \eta_{p(s)}(x)}{1+\varepsilon_{1}}-\varepsilon m+1\right) \\
& \quad-\operatorname{ord}\left(\xi_{t}(x)-\frac{\xi_{s}(x)+\varepsilon_{1} \eta_{p(s)}(x)}{1+\varepsilon_{1}}-\varepsilon x^{m}\right) \\
& \leq \operatorname{ord}\left(\xi_{t}(x)-\frac{\xi_{s}(x)+\varepsilon_{1} \eta_{p(s)}(x)}{1+\varepsilon_{1}}-\varepsilon x^{m}\right) \\
& \quad-\operatorname{ord}\left(\xi_{t}(x)-\frac{\xi_{s}(x)+\varepsilon_{1} \eta_{p(s)}(x)}{1+\varepsilon_{1}}-\varepsilon x^{m-1}\right),
\end{aligned}
$$

$s, t=1, \ldots, n$.

In turn (39) and the assumption $k \geq 2$ yield

$$
d_{i}^{2} \geq \sum_{q \in T_{i} \cap T\left(\psi_{k}(Y)\right)}\left(\operatorname{mt}\left(C_{i}, q\right)\right)^{2} \geq \frac{k}{k+1} \sum_{q \in T_{i}}\left(\operatorname{mt}\left(C_{i}, q\right)\right)^{2} \geq \frac{2}{3} \sum_{q \in T_{i}}\left(\operatorname{mt}\left(C_{i}, q\right)\right)^{2} .
$$

The final case in the proof of Lemma 11 is that of $k=1$ being defined by (17). This yields, in particular, that $C_{i}$ is singular at $z$. Indeed, $C_{i}$ is supposed to contain three points of $T\left(Y_{c l}\right)$, and if it were nonsingular, the third point in $T\left(C_{i}\right) \cap T\left(Y_{c l}\right)$ would have moved under transformation $\psi_{2}$ which contradicts the assumption $k=1$. Next, due to $T_{i}=T\left(C_{i}\right) \cap T\left(Y_{c l}\right) \subset T\left(C_{i}\right) \cap T\left(\psi_{2}\left(C_{i}\right)\right)$, we obtain

$$
\sum_{q \in T_{i}}\left(\operatorname{mt}\left(C_{i}, q\right)\right)^{2} \leq\left(C_{i} \cdot \psi_{2}\left(C_{i}\right)\right)_{z} .
$$

Let $c(x, y)=0$ be an equation of $C_{i}$ in an affine neighborhood of the point $z=(0,0)$.

Then

$$
\begin{gathered}
\left(C_{i} \cdot \psi_{2}\left(C_{i}\right)\right)_{z}=\left(c(x, y) \cdot c\left(x, y+\varepsilon x^{2}\right)\right)_{z} \\
=\left(c(x, y) \cdot\left(c\left(x, y+\varepsilon x^{2}\right)-c(x, y)\right)\right)_{z} \leq\left(c(x, y) \cdot\left(x^{2} \frac{\partial c}{\partial y}(x, y)\right)\right)_{z} \\
=2(c(x, y) \cdot x)_{z}+\left(c(x, y) \cdot \frac{\partial c}{\partial y}(x, y)\right)_{z} .
\end{gathered}
$$

On the other hand, for small $|\varepsilon|$, in some neighborhood $U$ of $z$, one has by $[8]$, Theorem 2(2),

$$
\begin{aligned}
d_{i}^{2} & \geq\left(C_{i} \cdot \psi_{1} C_{i}\right)_{U} \geq\left(c(x, y) \cdot\left(x \frac{\partial c}{\partial y}(x, y)\right)\right)_{z} \\
& =(c(x, y) \cdot x)_{z}+\left(c(x, y) \cdot \frac{\partial c}{\partial y}(x, y)\right)_{z} .
\end{aligned}
$$

Here $\partial c / \partial y(0,0)=0$, since $C_{i}$ is singular at $z$, and hence

$$
\left(c(x, y) \cdot \frac{\partial c}{\partial y}(x, y)\right)_{z} \geq(c(x, y) \cdot x)_{z} .
$$

Combining (41)-(44), one easily derives (34). 


\section{ANAlytic AND TOPOlOGiCAl ORDER OF A SINGUlar POINT. CuRves With PRESCRIBED SINGULARITIES}

\subsection{Plane curves with prescribed singularities.}

Theorem 1. Let $\left(C_{i}, z_{i}\right), i=1, \ldots, r$, be reduced plane curve germs with isolated singular points $z_{1}, \ldots, z_{r}$, respectively.

(1) If

$$
\left[\sqrt{\frac{3}{2} \sum_{i=1}^{r} M_{2}\left(Z^{s}\left(C_{i}, z_{i}\right)\right)}+\frac{\sum_{i=1}^{r} \operatorname{deg} Z^{s}\left(C_{i}, z_{i}\right)}{\sqrt{3 \sum_{i=1}^{r} M_{2}\left(Z^{s}\left(C_{i}, z_{i}\right)\right) / 2}}\right] \leq d+1,
$$

then there exists an irreducible plane curve $C$ of degree $d$ having $r$ singular points topologically equivalent to $\left(C_{1}, z_{1}\right), \ldots,\left(C_{r}, z_{r}\right)$, respectively, as its only singularities.

(2) If

$$
\left[\sqrt{\frac{3}{2} \sum_{i=1}^{r} M_{2}\left(Z^{a}\left(C_{i}, z_{i}\right)\right)}+\frac{\sum_{i=1}^{r} \operatorname{deg} Z^{a}\left(C_{i}, z_{i}\right)}{\sqrt{3 \sum_{i=1}^{r} M_{2}\left(Z^{a}\left(C_{i}, z_{i}\right)\right) / 2}}\right] \leq d+1,
$$

then there exists an irreducible plane curve $C$ of degree $d$ having $r$ singular points analytically equivalent to $\left(C_{1}, z_{1}\right), \ldots,\left(C_{r}, z_{r}\right)$, respectively, as its only singularities.

Furthermore, the germ at $C$ of the (topological or analytic) equisingular stratum in the space of curves of degree $d$ is T-smooth.

A particular case of one singularity is of special importance, since it will be used in constructing curves with prescribed singularities on arbitrary algebraic surfaces.

Definition 1. Given a reduced plane curve germ $(C, z)$, denote by $e^{s}(C, z)$ (resp., $e^{a}(C, z)$ ) the minimal degree $m$ of a plane curve $F$ having only one singular point $w$, which is topologically (resp., analytically) equivalent to $(C, z)$, and satisfying the condition

$$
H^{1}\left(\mathcal{J}_{Z / \mathbb{P}^{2}}(m-1)\right)=0,
$$

where $Z=Z^{e s}(F, w)$ (resp., $\left.Z=Z^{e a}(F, w)\right)$. We call the parameters $e^{s}$ and $s^{a}$ the topological and analytic order of a singular point.

It should be noted that $e^{s}, e^{a}$ introduced above differ from similar singular point invariants used in $6,13,14,21$. The present notion corresponds to strong transversality in the sense of [21]. More precisely,

Lemma 12. (1) Let $F$ be a plane curve as in Definition 1 and $L$ be a straight line which does not pass through $w$. Then the germ at $F$ of the family of curves of degree $m$, having in a neighborhood of $w$ a singular point topologically (resp., analytically) equivalent to $(C, z)$, is smooth of expected dimension, and transversally intersects the linear system

$$
\left\{G \in\left|\hat{\mathcal{O}}_{\mathbb{P}^{2}}(d)\right|: G \cap L=F \cap L\right\} .
$$

(2) Let $L \subset \mathbb{P}^{2}$ be a straight line. Then the set of $m$-tuples $\bar{z}$ of distinct points on $L$, such that there is a curve $F$ of degree $m$ as in Definition 1 , satisfying $F \cap L=\bar{z}$, is Zariski open in $\operatorname{Sym}^{m}(L)$. 
Proof. The first statement is equivalent to

$$
H^{1}\left(\mathcal{J}_{Z \cup(F \cap L) / \mathbb{P}^{2}}(m)\right)=0
$$

(see details in [5, 7, 20, where $Z$ is understood as in Definition 10, which follows from (47) and the exact sequence

$$
0=H^{1}\left(\mathcal{J}_{Z / \mathbb{P}^{2}}(m-1)\right) \rightarrow H^{1}\left(\mathcal{J}_{Z \cup(F \cap L) / \mathbb{P}^{2}}(m)\right) \rightarrow H^{1}\left(L, \mathcal{J}_{F \cap L}(m)\right)=0 .
$$

For the second statement take a curve $F$ as in Definition 1, which meets $L$ transversally, and the germ $M$ at $F$ of the family of curves of degree $m$ having in a neighborhood of $w$ a singular point topologically (resp., analytically) equivalent to $(C, z)$. Consider the map $G \in M \mapsto G \cap L \in \operatorname{Sym}^{m}(L)$. Then the preceding statement of the lemma means that this map is a submersion, and we are done.

Theorem 2. For any reduced plane curve germ $(C, z)$,

$$
\begin{aligned}
& e^{s}(C, z) \leq \sqrt{\frac{3}{2} M_{2}\left(Z^{s}(C, z)\right)}+\frac{\operatorname{deg} Z^{s}(C, z)}{\sqrt{3 M_{2}\left(Z^{s}(C, z)\right) / 2}}-1, \\
& e^{a}(C, z) \leq \sqrt{\frac{3}{2} M_{2}\left(Z^{a}(C, z)\right)}+\frac{\operatorname{deg} Z^{a}(C, z)}{\sqrt{3 M_{2}\left(Z^{a}(C, z)\right) / 2}}-1 .
\end{aligned}
$$

The hypotheses of Theorems 1 , 2 can be translated into more familiar singularity invariants.

Theorem 3. Let $\left(C_{i}, z_{i}\right), i=1, \ldots, r$, and $(C, z)$ be plane curve germs with isolated singular points $z_{1}, \ldots, z_{r}$ and $z$, respectively. Denote by $n, k$ and $t$ the number of nodes, cusps and ordinary triple points, respectively, among $\left(C_{1}, z_{1}\right), \ldots,\left(C_{r}, z_{r}\right)$.

(1) If

$$
6 n+10 k+\frac{169}{6} t+\frac{25}{3} u+\frac{27}{2} \sum_{\left(C_{i}, z_{i}\right) \neq A_{1}, A_{2}, D_{4}} \delta\left(C_{i}, z_{i}\right) \leq d^{2}-2 d+3,
$$

where $u$ is the number of points of type $A_{2 m}, m \geq 2$, among $\left(C_{i}, z_{i}\right), i=1, \ldots, r$, then there exists an irreducible curve of degree $d$ having $r$ singular points topologically equivalent to $\left(C_{1}, z_{1}\right), \ldots,\left(C_{r}, z_{r}\right)$, respectively, as its only singularities.

(2) If

(51) $6 n+10 k+\frac{169}{6} t+\sum_{\left(C_{i}, z_{i}\right) \neq A_{1}, A_{2}, D_{4}} \frac{\left(5 \mu\left(C_{i}, z_{i}\right)+3 \delta\left(C_{i}, z_{i}\right) / 2\right)^{2}}{3 \mu\left(C_{i}, z_{i}\right)+3 \delta\left(C_{i}, z_{i}\right) / 2} \leq d^{2}-2 d+3$,

then there exists an irreducible curve of degree d having $r$ singular points analytically equivalent to $\left(C_{1}, z_{1}\right), \ldots,\left(C_{r}, z_{r}\right)$, respectively, as its only singularities.

(3) If $(C, z)$ is of type $A_{m}, m \geq 1$, then

$$
e^{s}(C, z)=e^{a}(C, z) \leq 2[\sqrt{m+5}] ;
$$

if $(C, z)$ is of type $D_{m}, m \geq 4$, then

$$
e^{s}(C, z)=e^{a}(C, z) \leq 2[\sqrt{m+7}]+1 ;
$$

if $(C, z)$ is of type $E_{m}, m=6,7,8$, then

$$
e^{s}(C, z)=e^{a}(C, z)=\left[\frac{m+2}{2}\right]
$$


if $(C, z)$ is not simple, then

$$
\begin{gathered}
e^{s}(C, z) \leq \frac{9}{\sqrt{6}} \sqrt{\delta(C, z)}-1, \\
e^{a}(C, z) \leq \frac{5 \mu(C, z)+3 \delta(C, z) / 2}{\sqrt{3 \mu(C, z)+3 \delta(C, z) / 2}}-1 \leq 3 \sqrt{\mu(C, z)}-1 .
\end{gathered}
$$

Remark 5. We point out that, for specific singularities, invariants in the existence conditions can be reduced, for example, a curve with $n$ nodes and $k$ cusps exists if $2 n+4 k \leq d^{2}+O(d)$ [19], Theorem 4.1. We, however, have focused on obtaining a universal existence condition rather than an optimality of singularity invariants, although our results substantially improve all previously known general existence conditions [6, 14]. For example, since $\delta \leq 2 \mu / 3$ for singularities different from nodes, cusps and ordinary triple points, (51) yields the following weaker, but rather simpler sufficient existence condition for an irreducible plane curve with one or many singularities prescribed up to analytic equivalence:

$$
e^{a}(C, z) \leq 3 \sqrt{\mu(C, z)}-2(\text { as } \mu(C, z)>4), \quad \sum_{i=1}^{r} \mu\left(C_{i}, z_{i}\right) \leq \frac{1}{9}\left(d^{2}-2 d+3\right),
$$

which is much better than the previously known sufficient condition for the existence of an irreducible curve with singularities prescribed only up to topological equivalence (see [14, 6]):

$$
\begin{gathered}
e^{s}(C, z)<\min \left\{\sqrt{29 \mu(C, z)}+\frac{9}{2}, \sqrt{41.4 \mu(C, z)}-2\right\}, \\
\sum_{i=1}^{r} \mu\left(C_{i}, z_{i}\right) \leq \frac{1}{46}(d+2)^{2} .
\end{gathered}
$$

3.2. Proof of Theorem 11. Consider, first, the case of topological equivalence of singular points. By Lemma 2, without loss of generality, we can suppose that $Z^{s}\left(C_{i}, z_{i}\right)$ is a generic element of $\operatorname{Def}\left(Z^{s}\left(C_{i}, z_{i}\right)\right), i=1, \ldots, r$.

Inequality 45 means by Proposition 10 that

$$
H^{1}\left(\mathcal{J}_{Z / \mathbb{P}^{2}}(d-1)\right)=0,
$$

where $Z=Z^{s}\left(C_{1}, z_{1}\right) \cup \ldots \cup Z^{s}\left(C_{r}, z_{r}\right)$. Introduce the schemes

- $Z \cup\{z\}$, where $z \in \mathbb{P}^{2} \backslash\left\{z_{1}, \ldots, z_{r}\right\}$;

- $Z^{(i)}=Z^{s}\left(C_{1}, z_{1}\right) \cup \ldots \cup Z^{s}\left(C_{i-1}, z_{i-1}\right) \cup Z_{1}^{s}\left(C_{i}, z_{i}\right) \cup Z^{s}\left(C_{i+1}, z_{i+1}\right) \cup \ldots \cup$ $Z^{s}\left(C_{r}, z_{r}\right), i=1, \ldots, r$.

We claim that

$$
\begin{gathered}
H^{1}\left(\mathcal{J}_{Z \cup\{z\} / \mathbb{P}^{2}}(d)\right)=0, \quad z \in \mathbb{P}^{2} \backslash Z, \\
H^{1}\left(\mathcal{J}_{Z^{(i)} / \mathbb{P}^{2}}(d)\right)=0, \quad i=1, \ldots, r .
\end{gathered}
$$

Indeed, let $L_{0}$ be a generic straight line through $z$, and $L_{i}$ be a generic straight line through $z_{i}$. Then

$$
\operatorname{deg}\left(L_{0} \cap Z^{(0)}(z)\right)=\operatorname{deg}\{z\}=1 \leq d+1 \quad \Longrightarrow \quad H^{1}\left(L_{0}, \mathcal{J}_{z}(d)\right)=0 .
$$

In view of (52),

$$
H^{1}\left(L_{i}, \mathcal{J}_{L_{i} \cap Z}(d-1)\right)=0 \quad \Longrightarrow \quad \operatorname{deg}\left(L_{i} \cap Z^{s}\left(C_{i}, z_{i}\right)\right) \leq d ;
$$


hence

$$
\operatorname{deg}\left(L_{i} \cap Z_{1}^{s}\left(C_{i}, z_{i}\right)\right) \leq d+1 \Longrightarrow H^{1}\left(L_{i}, \mathcal{J}_{Z^{(i)}}(d)\right)=0 .
$$

Then, using the last $h^{1}$-vanishing statement, the relation $Z_{1}^{s}\left(C_{i}, z_{i}\right): L_{i}=Z^{s}\left(C_{i}, z_{i}\right)$ coming from Lemmas 2.14, 2.15 of [6], and the exact sequences

$$
\begin{gathered}
\quad 0 \rightarrow \mathcal{J}_{Z / \mathbb{P}^{2}}(d-1) \rightarrow \mathcal{J}_{Z \cup\{z\} / \mathbb{P}^{2}}(d) \rightarrow \mathcal{J}_{z}(d) \rightarrow 0, \\
0 \rightarrow \mathcal{J}_{Z / \mathbb{P}^{2}}(d-1) \rightarrow \mathcal{J}_{Z^{(i)} / \mathbb{P}^{2}}(d) \rightarrow \mathcal{J}_{L_{i} \cap Z_{1}^{s}\left(C_{i}, z_{i}\right) / L_{i}}(d) \rightarrow 0,
\end{gathered}
$$

one can easily derive (53), (54) from (52).

By Lemma 3(2), the $h^{1}$-vanishing (54) implies that, for any $i=1, \ldots, r$, there exists a curve $D_{i} \in\left|\mathcal{J}_{Z / \mathbb{P}^{2}}(d)\right|$ such that $\left(D_{i}, z_{i}\right)$ is topologically equivalent to $\left(C_{i}, z_{i}\right), 1 \leq j \leq r$. Again by Lemma 3] 2 ), for a generic curve $D$ of the linear system $\lambda_{1} D_{1}+\ldots+\lambda_{r} D_{r}, \lambda_{1}, \ldots, \lambda_{r} \in \mathbb{C}$, the germs $\left(D, z_{i}\right)$ are topologically equivalent to $\left(C_{i}, z_{i}\right), i=1, \ldots, r$, respectively. Furthermore, we can suppose that $D$ is reduced. Let $w_{1}, \ldots, w_{m}$ be all singular points of $D$ outside $z_{1}, \ldots, z_{r}$. By virtue of (53), there exist curves $D_{1}^{\prime}, \ldots, D_{m}^{\prime} \in\left|\mathcal{J}_{Z / \mathbb{P}^{2}}(d)\right|$ such that $w_{j} \notin D_{j}^{\prime}, j=1, \ldots, m$. By Bertini's theorem, the singular locus of a generic curve $D^{\prime}$ of the linear system $\lambda D+\Lambda_{1}^{\prime} D_{1}^{\prime}+\ldots+\lambda_{m}^{\prime} D_{m}^{\prime}, \lambda, \lambda_{1}^{\prime}, \ldots, \lambda_{m}^{\prime} \in \mathbb{C}$, is $\left\{z_{1}, \ldots, z_{r}\right\}$, and $\left(D^{\prime}, z_{i}\right)$ is topologically equivalent to $\left(C_{i}, z_{i}\right), i=1, \ldots, r$.

Finally, we show that $D^{\prime}$ is irreducible. The above argument shows that the linear system $\left|\mathcal{J}_{Z / \mathbb{P}^{2}}(d)\right|$ has no fixed part. If all the curves in $\left|\mathcal{J}_{Z / \mathbb{P}^{2}}(d)\right|$ are reducible, then by Bertini's theorem a generic curve $D^{\prime} \in\left|\mathcal{J}_{Z / \mathbb{P}^{2}}(d)\right|$ splits into irreducible components, which all belong to a one-dimensional linear system. In particular,

$$
\operatorname{dim}\left|\mathcal{J}_{Z / \mathbb{P}^{2}}(d)\right| \leq d
$$

On the other hand, by (52),

$$
\operatorname{dim}\left|\mathcal{J}_{Z / \mathbb{P}^{2}}(d)\right|=\frac{d(d+3)}{2}-\operatorname{deg} Z
$$

Inequalities (3) and (45) yield

$$
\begin{gathered}
d+2>\sqrt{\frac{3}{2} M_{2}(Z)}+\frac{\operatorname{deg} Z}{\sqrt{3 M_{2}(Z) / 2}} \\
\geq \sqrt{\frac{3}{2} \operatorname{deg} Z}+\frac{\operatorname{deg} Z}{\sqrt{3 \operatorname{deg} Z / 2}}=\frac{7}{\sqrt{6}} \sqrt{\operatorname{deg} Z} .
\end{gathered}
$$

Hence

$$
\begin{gathered}
\operatorname{dim}\left|\mathcal{J}_{Z / \mathbb{P}^{2}}(d)\right|-d=\frac{d(d+1)}{2}-\operatorname{deg} Z \\
>\frac{13}{12} \operatorname{deg} Z-\frac{15}{2 \sqrt{6}} \sqrt{\operatorname{deg} Z}+1 \geq 0
\end{gathered}
$$

as $\operatorname{deg} Z \geq 6$, and we are done, since the remaining cases of one node or one cusp are obviously covered by (45).

The case of the analytic equivalence of singular points can be treated in the same way when using Lemmas 44(2) and 5 instead of Lemma [3(2).

Finally, we note that, by construction, $Z^{s}\left(D, z_{i}\right)=Z^{s}\left(C_{i}, z_{i}\right)$ in the first case of the theorem, and $Z^{a}\left(D, z_{i}\right)=Z^{a}\left(C_{i}, z_{i}\right)$ in the second case, $i=1, \ldots, r$. In view of

$$
I^{s}\left(D, z_{i}\right) \subset I^{e s}\left(D, z_{i}\right), \quad I^{a}\left(D, z_{i}\right) \subset I^{e a}\left(D, z_{i}\right), \quad i=1, \ldots, r,
$$


(52) implies

$$
H^{1}\left(\mathcal{J}_{Z^{\prime} / \mathbb{P}^{2}}(d)\right)=0,
$$

with the zero-dimensional scheme $Z^{\prime}$ defined at points $z_{1}, \ldots, z_{r}$ by the ideals $I^{e s}\left(D, z_{i}\right)$ or $I^{e a}\left(D, z_{i}\right)$, respectively to the case considered. In turn the last $h^{1}$ vanishing means the $T$-smoothness of the topological or analytic equisingular stratum at $D$ in the space of curves of degree $d$ (see [5] 6] [7, 20, for details).

3.3. Proof of Theorem 3. The case $d \leq 3$ is trivial, so we assume that $d \geq 4$.

(1) If there are no nodes and cusps among $\left(C_{i}, z_{i}\right), i=1, \ldots, r$, then for an ordinary triple point $\left(C_{i}, z_{i}\right)$

$$
\operatorname{deg} Z^{s}\left(C_{i}, z_{i}\right)=6, \quad M_{2}\left(Z^{s}\left(C_{i}, z_{i}\right)\right)=9,
$$

for a point of type $A_{2 m}, m \geq 2$, by (3) and Lemma 7 ,

$$
\operatorname{deg} Z^{s}\left(C_{i}, z_{i}\right)=3 \delta\left(C_{i}, z_{i}\right)+2, \quad M_{2}\left(Z^{s}\left(C_{i}, z_{i}\right)\right)=4 \delta\left(C_{i}, z_{i}\right)+2,
$$

and for the rest of the germs,

$$
\operatorname{deg} Z^{s}\left(C_{i}, z_{i}\right) \leq 3 \delta\left(C_{i}, z_{i}\right), \quad M_{2}\left(Z^{s}\left(C_{i}, z_{i}\right)\right) \leq 4 \delta\left(C_{i}, z_{i}\right) .
$$

Hence

$$
\begin{aligned}
& (55) \quad\left(\sqrt{\frac{3}{2} \sum_{i=1}^{r} M_{2}\left(Z^{s}\left(C_{i}, z_{i}\right)\right)}+\frac{\sum_{i=1}^{r} \operatorname{deg} Z^{s}\left(C_{i}, z_{i}\right)}{\sqrt{3 \sum_{i=1}^{r} M_{2}\left(Z^{s}\left(C_{i}, z_{i}\right)\right) / 2}}\right)^{2} \\
& \leq \frac{\left(9 \sum_{\left(C_{i}, z_{i}\right) \neq D_{4}} \delta\left(C_{i}, z_{i}\right)+39 t / 2+5 u\right)^{2}}{6 \sum_{\left(C_{i}, z_{i}\right) \neq D_{4}} \delta\left(C_{i}, z_{i}\right)+27 t / 2+3 u} \leq \frac{27}{2} \sum_{\left(C_{i}, z_{i}\right) \neq D_{4}} \delta\left(C_{i}, z_{i}\right)+\frac{169}{6} t+\frac{25}{3} u .
\end{aligned}
$$

Thus, (50) implies (45), and we are done.

Assume that $n+k>0$. Put

$$
s=\max \left\{t \geq 2: \sum_{i=1}^{t-2}(d-i)+1 \leq 3 n+5 k\right\} .
$$

Observe that by (50), $s \leq d-1$. Inequalities (50), (55) also yield

$$
\begin{gathered}
\sqrt{\frac{3}{2} \sum_{\left(C_{i}, z_{i}\right) \neq A_{1}, A_{2}} M_{2}\left(Z^{s}\left(C_{i}, z_{i}\right)\right)}+\frac{\sum_{\left(C_{i}, z_{i}\right) \neq A_{1}, A_{2}} \operatorname{deg} Z^{s}\left(C_{i}, z_{i}\right)}{\sqrt{3 \sum_{\left(C_{i}, z_{i}\right) \neq A_{1}, A_{2}} M_{2}\left(Z^{s}\left(C_{i}, z_{i}\right)\right) / 2}} \\
\leq \sqrt{\frac{27}{2} \sum_{\left(C_{i}, z_{i}\right) \neq A_{1}, A_{2}, D_{4}} \delta\left(C_{i}, z_{i}\right)+\frac{169}{6} t+\frac{25}{3} u} \\
\leq \sqrt{d^{2}-2 d+3-6 n-10 k} \leq \sqrt{d^{2}-2 d+3-2 \sum_{i=1}^{s-2}(d-i)-2} \\
=\sqrt{d^{2}-2(s-1) d+(s-1)(s-2)+1} \leq d-s+1 ;
\end{gathered}
$$

hence, by Proposition 10

$$
H^{1}\left(\mathcal{J}_{Z^{\prime} / \mathbb{P}^{2}}(d-s-1)\right)=0,
$$

where $Z^{\prime}$ is the part of $Z$ without nodes and cusps. Then we derive (45), and thereby the first statement of Theorem 3 , from the following lemma. 
Lemma 13. Let $L \subset \mathbb{P}^{2}$ be a straight line, $X, Y \subset \mathbb{P}^{2}$ be zero-dimensional schemes such that $L \cap Y=\emptyset, X \cap Y=\emptyset$, and $X=X^{(1)} \cup X^{(2)} \cup X^{(3)} \cup X^{(4)}$, where $X^{(1)}$ is a union of schemes of degree 1 (i.e., locally defined by the maximal ideal), $X^{(2)}$ is a union of schemes of degree 2 (i.e., locally defined by ideals like $\left.\left\langle y, x^{2}\right\rangle\right), X^{(3)}$ is a union of schemes from $\operatorname{Def}\left(Z^{s}\right.$ (node)) (i.e., locally defined by the square of the maximal ideal), and $Z^{(4)}$ is a union of schemes from $\operatorname{Def}\left(Z^{s}(\right.$ cusp $)$ ) (i.e., locally defined by ideals like $\left.\left\langle y^{2}, y x^{2}, x^{3}\right\rangle\right)$. Assume that

(i) $H^{1}\left(\mathcal{J}_{Y / \mathbb{P}^{2}}(d-s-1)\right)=0$, where

$$
s=\max \left\{t \geq 2: \sum_{i=1}^{t-2}(d-i)+1 \leq \operatorname{deg} X\right\} \quad \text { and } \quad s \leq d-1
$$

(ii) $X^{(4)} \cap L=\emptyset, \operatorname{deg}(X \cap L) \leq d$;

(iii) the components of $X$ which do not meet $L$ are placed in $\mathbb{P}^{2} \backslash(Y \cup L)$ in a general position.

Then

$$
H^{1}\left(\mathcal{J}_{X \cup Y / \mathbb{P}^{2}}(d-1)\right)=0 .
$$

Proof of Lemma 13 We perform induction on $s$, using the so-called "Horace method" [12] (see also [6] and Step 4 in the proof of Proposition [10 above).

If $s=2$, then $\operatorname{deg} X \leq d-1$. We specialize all the components of $X$ on the line $L$ with maximal possible intersection with $L$. First, we note that (56) for the specialized scheme $X$ implies the same relation for the original $X$ due to the semicontinuity of cohomology. Second,

$$
\operatorname{deg}((X: l) \cap L) \leq \operatorname{deg}(X \cap L) \leq \operatorname{deg} X \leq d-1,
$$

and $X \subset L^{2}$. Then (56) follows from the two exact sequences

$$
\begin{gathered}
0=H^{1}\left(\mathcal{J}_{Y / \mathbb{P}^{2}}(d-3)\right) \rightarrow H^{1}\left(\mathcal{J}_{(X: L) \cup Y / \mathbb{P}^{2}}(d-2)\right) \rightarrow H^{1}\left(L, \mathcal{J}_{(X: L) \cap L}(d-2)\right)=0 \\
H^{1}\left(\mathcal{J}_{(X: L) \cup Y / \mathbb{P}^{2}}(d-2)\right) \rightarrow H^{1}\left(\mathcal{J}_{X \cup Y / \mathbb{P}^{2}}(d-1)\right) \rightarrow H^{1}\left(L, \mathcal{J}_{X \cap L}(d-1)\right)=0 .
\end{gathered}
$$

Let $s \geq 3$ and $d-1 \leq \operatorname{deg}(X \cap L) \leq d$. Then $H^{1}\left(L, \mathcal{J}_{X \cap L}(d-1)\right)=0$, and (57) reduces (56) to $H^{1}\left(\mathcal{J}_{(X: L) \cup Y / \mathbb{P}^{2}}(d-2)\right)=0$, which holds by the induction assumption. Indeed, one can easily check that $X: L$ is the union of zero-dimensional schemes of the same four kinds as $X$, the intersection $(X: L) \cap L$ is the union of schemes of degree 1 , and $\operatorname{deg}((X: L) \cap L)<\operatorname{deg}(X \cap L) \leq d$. At last, since

$$
\sum_{i=1}^{s-1}(d-i)+1>\operatorname{deg} X
$$

then

so

$$
\sum_{i=1}^{s-2}(d-1-i)+1>\operatorname{deg} X-(d-1) \geq \operatorname{deg}(X: L),
$$

$$
s-1 \geq \max \left\{t \geq 2: \sum_{i=1}^{t-2}(d-1-i)+1 \leq \operatorname{deg} X\right\} .
$$

Let $s \geq 3$ and $\operatorname{deg}(X \cap L) \leq d-2$. If all the components of $X$ are specialized on $L$, then we complete the proof as in the case $s=2$. If there are components of 
$X$ out of $L$, we specialize some of them on $L$ keeping three rules:

- $\operatorname{deg}(X \cap L) \leq d$,

- if $\operatorname{deg}(X \cap L) \leq d-2$, then a component $X^{\prime}$ of degree 2 of $X$ is specialized on $L$ so that $\operatorname{deg}\left(X^{\prime} \cap L\right)=2$ (with tangency),

- if $\operatorname{deg}(X \cap L) \leq d-3$, then a component $X^{\prime} \in \operatorname{Def}$ (cusp) is specialized on $L$ so that $\operatorname{deg}\left(X^{\prime} \cap L\right)=3$ (with tangency).

If we end up with $d-1 \leq \operatorname{deg}(X \cap L) \leq d$, then notice that the components of $X: L$ meeting $L$ are of degree $\leq 2$ or belong to $\operatorname{Def}($ node), and $\operatorname{deg}((X: L) \cap L) \leq$ $\operatorname{deg}(X \cap L)-1 \leq d-1$; this allows us to complete the proof as in the preceding paragraph. If we end up with $\operatorname{deg}(X \cap L) \leq d-2$, and all components of $X$ specialized on $L$ so that the components from Def(cusp) are tangent to $L$, then $X \subset L^{2}$ and we complete the proof as in the case $s=2$.

(2) If there are no nodes and cusps among $\left(C_{i}, z_{i}\right), i=1, \ldots, r$, then by (3) and Lemma 7.

$\operatorname{deg} Z^{a}\left(C_{i}, z_{i}\right) \leq 2 \mu\left(C_{i}, z_{i}\right), M_{2}\left(Z^{a}\left(C_{i}, z_{i}\right)\right) \leq 2 \mu\left(C_{i}, z_{i}\right)+\delta\left(C_{i}, z_{i}\right),\left(C_{i}, z_{i}\right) \neq D_{4}$.

Hence

$$
\begin{gathered}
\left(\sqrt{\frac{3}{2} \sum_{i=1}^{r} M_{2}\left(Z^{a}\left(C_{i}, z_{i}\right)\right)}+\frac{\sum_{i=1}^{r} \operatorname{deg} Z^{a}\left(C_{i}, z_{i}\right)}{\sqrt{3 \sum_{i=1}^{r} M_{2}\left(Z^{a}\left(C_{i}, z_{i}\right)\right) / 2}}\right)^{2} \\
l e \frac{\left(\sum_{\left(C_{i}, z_{i}\right) \neq D_{4}}\left(5 \mu\left(C_{i}, z_{i}\right)+3 \delta\left(C_{i}, z_{i}\right) / 2\right)+39 t / 2\right)^{2}}{\sum_{\left(C_{i}, z_{i}\right) \neq D_{4}}\left(3 \mu\left(C_{i}, z_{i}\right)+3 \delta\left(C_{i}, z_{i}\right) / 2\right)+27 t / 2} \\
\leq \sum_{\left(C_{i}, z_{i}\right) \neq D_{4}} \frac{\left(5 \mu\left(C_{i}\right)+3 \delta\left(C_{i}, z_{i}\right) / 2\right)^{2}}{3 \mu\left(C_{i}, z_{i}\right)+3 \delta\left(C_{i}, z_{i}\right) / 2}+\frac{169}{6} t .
\end{gathered}
$$

Thus, (51) implies (46), and we are done.

If $n+k>0$ we prove the second statement of Theorem 3 in the same way as in the first part of the proof.

(3) Estimates for $e^{s}(C, z), e^{a}(C, z)$ (increased by 1 in view of condition (47)), where $(C, z)$ is of type $A_{k}, D_{k}$, are taken from [14. For singularities $E_{k}, k=6,7,8$, one has classically known curves of degree $d=4,4,5$, respectively. Relation (47) in these cases holds by [17], Theorem 1.1(2), under the condition $k<3 d-3$. The other estimates follow from (3), Lemma 7) Theorem 2, and the inequality $\delta(C, z) \leq 2 / 3 \cdot \mu(C, z)$ for a nonsimple singular point.

3.4. Curves with prescribed singularities on algebraic surfaces. Let $\Sigma$ be a smooth algebraic surface and $D \subset \Sigma$ be a divisor with $\operatorname{dim}|D|>0$. To obtain a criterion for the existence of a curve $C \in|D|$ with prescribed singularities, we combine [21, Theorem 1, which basically reduces the problem to $h^{1}$-vanishing for the ideal sheaf of a zero-dimensional subscheme of $\Sigma$ defined by $e^{s}$ or $e^{a}$-powers of local maximal ideals, [13, Theorem 2.1, which provides a numerical sufficient condition for the above $h^{1}$-vanishing, and Theorem 3(3) with upper bounds to $e^{s}$ and $e^{a}$.

Theorem 4. Let $\Sigma$ be a smooth projective surface, $D$ a divisor on $\Sigma$ with $D-K_{\Sigma}$ nef, and $L \subset \Sigma$ a very ample divisor. Let $\left(C_{1}, z_{1}\right), \ldots,\left(C_{r}, z_{r}\right)$ be reduced singular germs of plane curves, among them $n$ nodes and $k$ cusps. 
(1) If

$$
\begin{gathered}
18 n+32 k+27 \sum_{\delta\left(C_{i}, z_{i}\right)>1} \delta\left(C_{i}, z_{i}\right) \leq\left(D-K_{\Sigma}-L\right)^{2}, \\
\left(D-L-K_{\Sigma}\right) L>\frac{9}{\sqrt{6}} \max _{1 \leq i \leq r} \sqrt{\delta\left(C_{i}, z_{i}\right)}+1,
\end{gathered}
$$

and, for any irreducible curve $B$ with $B^{2}=0$ and $\operatorname{dim}|B|_{a}>0$,

$$
\frac{9}{\sqrt{6}} \max _{1 \leq i \leq r} \sqrt{\delta\left(C_{i}, z_{i}\right)}<\left(D-K_{\Sigma}-L\right) B+1,
$$

then there exists an irreducible curve $C \in|D|$ with $r$ singular points topologically equivalent to $\left(C_{1}, z_{1}\right), \ldots,\left(C_{r}, z_{r}\right)$, respectively, as its only singularities.

(2) If

$$
\begin{gathered}
18 n+32 k+18 \sum_{\mu\left(C_{i}, z_{i}\right)>2} \mu\left(C_{i}, z_{i}\right) \leq\left(D-K_{\Sigma}-L\right)^{2}, \\
\left(D-L-K_{\Sigma}\right) L>3 \max _{1 \leq i \leq r} \sqrt{\mu\left(C_{i}, z_{i}\right)}+1
\end{gathered}
$$

and, for any irreducible curve $B$ with $B^{2}=0$ and $\operatorname{dim}|B|_{a}>0$,

$$
3 \max _{1 \leq i \leq r} \sqrt{\mu\left(C_{i}, z_{i}\right)}<\left(D-K_{\Sigma}-L\right) B+1,
$$

then there exists an irreducible curve $C \in|D|$ with $r$ singular points analytically equivalent to $\left(C_{1}, z_{1}\right), \ldots,\left(C_{r}, z_{r}\right)$, respectively, as its only singularities.

Here $|B|_{a}$ means the family of curves algebraically equivalent to $B$.

Proof. Step 1. Since $e^{s}($ node $)=2, e^{s}(\operatorname{cusp})=3$, and by Theorem 313$)$,

$$
e^{s}\left(C_{i}, z_{i}\right)+1 \leq \frac{9}{\sqrt{6}} \sqrt{\delta\left(C_{i}, z_{i}\right)} \quad \text { as } \quad \delta\left(C_{i}, z_{i}\right)>1,
$$

(58) and (60) imply

$$
\begin{gathered}
2 \sum_{i=1}^{r}\left(e^{s}\left(C_{i}, z_{i}\right)+1\right)^{2} \leq\left(D-K_{\Sigma}-L\right)^{2}, \\
\left(D-K_{\Sigma}-L\right) B>\max _{1 \leq i \leq r} e^{s}\left(C_{i}, z_{i}\right),
\end{gathered}
$$

for curves $B$ as in (60). By 13, Theorem 2.1, this yields

$$
H^{1}\left(\Sigma, \mathcal{J}_{Z}(D-L)\right)=0
$$

where $Z \subset \Sigma$ is a zero-dimensional scheme concentrated at generic points $w_{1}, \ldots, w_{r}$ in $\Sigma$ and defined by the ideals $\left(\mathfrak{m}_{w_{i}}\right)^{e^{s}\left(C_{i}, z_{i}\right)}, i=1, \ldots, r$.

Step 2. Let $w_{0}$ be a generic point in $\Sigma \backslash\left\{w_{1}, \ldots, w_{r}\right\}$, and let $w$ be any point in $\Sigma \backslash\left\{w_{1}, \ldots, w_{r}, w\right\}$. Since $L$ is very ample, there is a nonsingular connected curve in $|L|$ (which we further denote by $L$ as well) which passes through $w, w_{0}$ and, perhaps, through one of $w_{1}, \ldots, w_{r}$. In the exact sequence

$$
0=H^{1}\left(\Sigma, \mathcal{J}_{Z}(D-L)\right) \rightarrow H^{1}\left(\Sigma, \mathcal{J}_{Z \cup\left\{w, w_{0}\right\}}(D)\right) \rightarrow H^{1}\left(L, \mathcal{J}_{(Z \cap L) \cup\left\{w, w_{0}\right\}}(D)\right),
$$

the latter term vanishes, since by (59),

$$
\operatorname{deg} \mathcal{J}_{(Z \cap L) \cup\left\{w, w_{0}\right\}}(D) \geq D L-2-\max _{1 \leq i \leq r} e^{s}\left(C_{i}, z_{i}\right)>L^{2}+L K_{\Sigma}=2 g(L)-2 .
$$


Hence

$$
H^{1}\left(\Sigma, \mathcal{J}_{Z \cup\left\{w, w_{0}\right\}}(D)\right)=0 .
$$

In particular, there exists a curve in $\left|\mathcal{J}_{Z \cup\left\{w_{0}\right\}}(D)\right|$ which does not pass through $w$. Since $w_{0}$ is generic and $w$ is any point outside $w_{0}, \ldots, w_{r}$, by Bertini's theorem a generic curve $D_{0} \in\left|\mathcal{J}_{Z \cup\left\{w_{0}\right\}}(D)\right|$ is nonsingular outside $w_{1}, \ldots, w_{r}$, and this linear system has no fixed part. In addition, $D_{0}$ is irreducible. Indeed, otherwise, by Bertini's theorem, $D_{0}$ and all close curves in $\left|\mathcal{J}_{Z \cup\left\{w_{0}\right\}}(D)\right|$ would split into variable components which belong to the same one-dimensional algebraic family. However, this contradicts the fact that $D_{0}$ is nonsingular at the fixed point $w_{0}$.

Step 3. For any $i=1, \ldots, r$, define a zero-dimensional scheme $Z_{i}$ which coincides with $Z$ at $w_{j}, 1 \leq j \leq r, j \neq i$, and is given by the ideal $\left(\mathfrak{m}_{w_{i}}\right)^{e^{s}\left(C_{i}, z_{i}\right)+1}$ at $w_{i}$. We claim that

$$
H^{1}\left(\Sigma, \mathcal{J}_{Z_{i}}(D)\right)=0 .
$$

Indeed, there exists a nonsingular curve in $|L|$ (which we again denote by $L$ for the sake of notation) which passes through $w_{i}$ and does not contain any point $w_{j}$, $j \neq i$. Then in the exact sequence

$$
0=H^{1}\left(\Sigma, \mathcal{J}_{Z}(D-L)\right) \rightarrow H^{1}\left(\Sigma, \mathcal{J}_{Z_{i}}(D)\right) \rightarrow H^{1}\left(L, \mathcal{J}_{Z_{i} \cap L}(D)\right),
$$

the last term vanishes since by (59),

$$
D L-\operatorname{deg}\left(Z_{i} \cap L\right) \geq D L-e^{s}\left(C_{i}, z_{i}\right)>L^{2}+L K_{\Sigma}=2 g(L)-2 .
$$

Step 4. Using (61), for any $i=1, \ldots, r$, we can find a curve $D_{i} \in\left|\mathcal{J}_{Z / \Sigma}(D)\right|$ which has an ordinary singular point of multiplicity $e^{s}\left(C_{i}, z_{i}\right)$ at $w_{i}$, and, in addition, in some fixed local coordinates in a neighborhood of $w_{i}$, the $e^{s}\left(C_{i}, z_{i}\right)$-jet at $w_{i}$ of a local equation of $D_{i}$ is a generi $\mathbb{A}^{s} e^{s}\left(C_{i}, z_{i}\right)$-form. Then, a generic curve $\widetilde{D}$ in the linear system spanned by $D_{0}, D_{1}, \ldots, D_{r}$, is irreducible, nonsingular outside $w_{1}, \ldots, w_{r}$, has an ordinary singular point of multiplicity $e^{s}\left(C_{i}, z_{i}\right)$ at $w_{i}, i=1, \ldots, r$, and, finally, the $e^{s}\left(C_{i}, z_{i}\right)$-jet at $w_{i}$ of a local equation of $\widetilde{D}$ in some fixed coordinates in a neighborhood of $w_{i}$ is a generic $e^{s}\left(C_{i}, z_{i}\right)$-form, $i=1, \ldots, r$.

Step 5. By Lemma12(2), for any $i=1, \ldots, r$, there is an affine curve $F_{i}$ of degree $e^{s}\left(C_{i}, z_{i}\right)$ with its only singular point topologically equivalent to $\left(C_{i}, z_{i}\right)$, and such that the leading form of the defining polynomial coincides with the $e^{s}\left(C_{i}, z_{i}\right)$-jet at $w_{i}$ of a local equation of $\widetilde{D}$. Now we apply [21], Theorem 1 , to deform $\widetilde{D}$ into the required curve $C \in|D|$ with prescribed singularities.

Namely, in the assertion of [21], Theorem 1, $S=S_{1}=S_{3}=S_{4}=S_{5}=\emptyset$, $S_{2}=\left\{w_{1}, \ldots, w_{r}\right\}$, the affine curves $F_{1}, \ldots, F_{r}$ serve as deformation models for the singular points of $\widetilde{D}$, so that these models are strongly transversal with respect to the topological equivalence of singular points in view of (47). Furthermore, the $h^{1}$-vanishing condition in [21], Theorem 1 , reads as

$$
H^{1}\left(\Sigma, \mathcal{J}_{\widetilde{Z}}(D)\right)=0
$$

where $\widetilde{Z}=\bigcup_{i} Z^{\text {es }}\left(\widetilde{D}, w_{i}\right)$, which immediately follows, say, from , since $Z_{i} \supset \widetilde{Z}$ for any $i=1, \ldots, r$.

\footnotetext{
${ }^{4}$ Here "generic" means that the object considered can be chosen arbitrarily in a Zariski open subset of the whole space of objects.
} 
Step 6. The second statement of Theorem 4 can be proven in the same way, when taking into account that, by Theorem $3(3), e^{a}\left(C_{i}, z_{i}\right) \leq 3 \sqrt{\mu\left(C_{i}, z_{i}\right)}-1$ as $\mu\left(C_{i}, z_{i}\right)>2$.

\section{Analytic order of a CRItical Point}

Let $f \in \hat{\mathcal{O}}_{\mathbb{C}^{2}, 0}, \mu(f)<\infty$. Denote by $e^{a}(f)$ the minimal degree of a polynomial $p \in \mathbb{C}[x, y]$ right equivalent to $f$ at 0 , i.e., there is a local diffeomorphism $\varphi$ : $\left(\mathbb{C}^{2}, 0\right) \rightarrow\left(\mathbb{C}^{2}, 0\right)$ such that $p=f \circ \varphi$ in a neighborhood of the origin.

Theorem 5. If $f$ is of type $A_{m}, m \geq 1$, then

$$
e^{a}(f) \leq 2[\sqrt{m+5}]-1
$$

if $f$ is of type $D_{m}, m \geq 4$, then

$$
e^{a}(f) \leq 2[\sqrt{m+7}]
$$

if $f$ is of type $E_{m}, m=6,7,8$, then

$$
e^{a}(f)=\left[\frac{m+2}{2}\right]
$$

if $f$ is not simple, then

$$
e^{a}(f)<\frac{4}{\sqrt{3}} \sqrt{3 \mu(f)-2 \cdot \operatorname{mt}(f)+2}-1 .
$$

Proof. Since the case of a simple germ $f$ coincides with the case of a simple curve germ $\{f=0\}$, we take corresponding bounds from Theorem 3(3) (note only that here there is no need to increase the estimates from 14 by 1 , since (47) is not required). Let $f$ be not simple. We claim that

$$
\operatorname{ord}_{1}^{a n}(Z(f))<\frac{4}{\sqrt{3}} \sqrt{3 \mu(f)-2 \cdot \operatorname{mt}(f)+2}-1 .
$$

Indeed, by (4), Lemma 7], and Proposition [10,

$$
\begin{aligned}
& \operatorname{ord}_{1}^{a n}\left(Z_{0}(f)\right) \leq \frac{\operatorname{dim} Z_{0}(f)}{\sqrt{3 M_{2}\left(Z_{0}(f)\right) / 2}}+\sqrt{\frac{3}{2} M_{2}\left(Z_{0}(f)\right)}-2 \\
& <\frac{4}{\sqrt{3}} \sqrt{\operatorname{deg} Z_{0}(f)}-2 \leq \frac{4}{\sqrt{3}} \sqrt{3 \mu(f)-2 \cdot \operatorname{mt}(f)+2}-2 .
\end{aligned}
$$

Hence there is a germ $g \in \hat{\mathcal{O}}_{\mathbb{C}^{2}, 0}$ right equivalent to $f$ such that

$$
H^{1}\left(\hat{\mathcal{O}} Z_{0}(g) / \mathbb{P}^{2}(m)\right)=0, \quad m=\left[\frac{4}{\sqrt{3}} \sqrt{3 \mu(f)-2 \cdot \operatorname{mt}(f)+2}\right]-2 .
$$

For a generic straight line $L$ intersecting $Z_{0}(g)$, we have

$$
Z(g): L=Z_{0}(g), \quad \operatorname{deg}(Z(g) \cap L) \leq \operatorname{mt} Z_{0}(g)+1 \leq \operatorname{deg} Z_{0}(g) \leq m ;
$$

hence

$$
0=H^{1}\left(\hat{\mathcal{O}}_{Z_{0}(g) / \mathbb{P}^{2}}(m)\right) \rightarrow H^{1}\left(\hat{\mathcal{O}}_{Z(g) / \mathbb{P}^{2}}(m+1)\right) \rightarrow H^{1}\left(L, \hat{\mathcal{O}}_{Z(g) \cap L}(m+1)\right)=0,
$$

which yields

$$
H^{1}\left(\hat{\mathcal{O}}_{Z(g) / \mathbb{P}^{2}}(m+1)\right)=0 .
$$

Then the surjectivity of the morphism

$$
H^{0}\left(\hat{\mathcal{O}}_{\mathbb{P}^{2}}(m+1)\right) \rightarrow H^{0}\left(\hat{\mathcal{O}}_{Z(g)}\right)=\hat{\mathcal{O}}_{\mathbb{C}^{2}, 0} / I(g)
$$


gives us a polynomial $p \in \mathbb{C}[x, y]$ of degree $\leq m+1$ such that $p-g \in I(g)$, and we are done by Lemma 6 2 ).

\section{Higher-Dimensional Case: Example}

Let $f:\left(\mathbb{C}^{n}\right) \rightarrow(\mathbb{C}, 0), n \geq 3$, be a germ of a holomorphic function with an isolated critical point, i.e., $\mu(f)<\infty$. As for $n=2$, we would like to estimate the minimal degree $d(f)$ of a polynomial $p \in \mathbb{C}\left[x_{1}, \ldots, x_{n}\right]$ right equivalent to $f$ at the origin. The classical bounds are

$$
\sqrt[n]{\mu(f)}+1 \leq d(f) \leq \mu(f)+1 .
$$

Analogously to the two-dimensional case we state

Conjecture 1. There exists a sequence of positive numbers $a_{n}, n \geq 3$, such that

$$
d(f) \leq a_{n} \sqrt[n]{\mu(f)}
$$

for any germ $f:\left(\mathbb{C}^{n}\right) \rightarrow(\mathbb{C}, 0)$ with $\mu(f)<\infty$.

This conjecture is elementary for $n=1$, and follows from Theorem 5 for $n=2$. It, in fact, implies similar bounds for isolated singular points of hypersurfaces in $\mathbb{P}^{n}$, and sufficient existence conditions for hypersurfaces of a given degree with prescribed isolated singularities.

To support Conjecture 10 we prove it in any dimension for the case of critical points of type $A_{k}, k \geq 1$.

Theorem 6. There is a sequence of positive numbers $a_{n}, n \geq 1$, such that

$$
d(f) \leq a_{n} \sqrt[n]{k}
$$

for any germ $f:\left(\mathbb{C}^{n}, 0\right) \rightarrow(\mathbb{C}, 0), n \geq 1$, of type $A_{k}, k \geq 1$.

Remark 6 . For $n=2$ one can produce explicit formulas for polynomials of degree $\leq a_{2} \sqrt{k}$ with a critical point of type $A_{k}$, like the classically known polynomial $\left(y-x^{m}\right)^{2}+y^{2 m}$ of degree $2 m$ with the critical point of type $A_{2 m^{2}-1}$ at the origin (see more examples in 9, 14]). We do not know similar formulas for $n \geq 3$, and provide an existence proof in the spirit of preceding sections.

Proof. For a germ $f:\left(\mathbb{C}^{n}, 0\right) \rightarrow(\mathbb{C}, 0)$ of type $A_{k}, k \geq 1$, introduce the zerodimensional schemes $Z_{0}(f)$ and $Z(f)$ defined at the origin by the ideals

$$
I_{0}(f)=\left\{g \in \hat{\mathcal{O}}_{\mathbb{C}^{n}, 0}: g, g_{x_{1}}, \ldots, g_{x_{n}} \in\left\langle f_{x_{1}}, \ldots, f_{x_{n}}\right\rangle\right\}, \quad I(f)=\mathfrak{m}_{0} \cdot I_{0}(f)
$$

(cf. section 1.4). Similar to the proof of Theorem 5 we conclude that

$$
d(f) \leq \operatorname{ord}_{1}^{a n}(Z(f)) \leq \operatorname{ord}_{1}^{a n}\left(Z_{0}(f)\right)+1 .
$$

We shall show that there is a sequence of positive numbers $b_{n}, n \geq 1$, such that

$$
\operatorname{ord}_{1}^{a n}\left(Z_{0}(f)\right) \leq b_{n} \sqrt[n]{k} .
$$

In the sequel we shall use an auxiliary statement.

Lemma 14. In the given notation:

(1) After a suitable linear coordinate change, the hypersurface germs $\left\{f_{x_{2}}=0\right\}$, $\ldots,\left\{f_{x_{n}}=0\right\}$ are nonsingular and intersect transversally along a nonsingular curve

$$
\gamma(t)=\left(x_{1}(t), \ldots, x_{n}(t)\right), \quad x_{1}(t)=t, x_{i}(t)=O\left(t^{2}\right), i=2, \ldots, n .
$$


The intersection multiplicity of $\gamma$ and $\left\{f_{x_{1}}=0\right\}$ at the origin is

$$
\left(\gamma \cdot f_{x_{1}}\right)_{0}=\mu(f)=k \text {. }
$$

(2) Introduce an ascending sequence of zero-dimensional schemes $Z_{m}, m \geq 1$, defined at the origin by the ideals

$$
I_{m}=\left\{g \in \hat{\mathcal{O}}_{\mathbb{C}^{n}, 0}:(\gamma \cdot g)_{0},\left(\gamma \cdot g_{x_{1}}\right)_{0}, \ldots,\left(\gamma \cdot g_{x_{n}}\right)_{0} \geq m\right\}, \quad m \geq 1 .
$$

Let $L \subset \mathbb{P}^{n}$ be a hyperplane such that $L \cap \mathbb{C}^{n}=\left\{x_{n}=0\right\}$ and $2 \leq(\gamma \cdot L)_{0}=m_{0} \leq k$. Assume that the function germ $\left.f\right|_{L}:(L, 0) \rightarrow(\mathbb{C}, 0)$ has an isolated critical point. Then

(i) $Z_{k}=Z_{0}(f)$,

(ii) $Z_{m} \cap L \subset Z_{0}\left(\left.f\right|_{L}\right)$ for all $m \leq k$,

(iii) $Z_{m}: L^{2} \subset Z_{m-m_{0}}$ if $m>m_{0}$, and $Z_{m} \subset L^{2}$ if $m \leq m_{0}$.

Proof of Lemma 14. For the first claim we observe that for suitable coordinates $f\left(x_{1}, \ldots, x_{n}\right)=x_{2}^{2}+\ldots+x_{n}^{2}+$ h.o.t.

Statement $2(\mathrm{i})$ becomes trivial when passing to local coordinates $y_{1}, \ldots, y_{n}$ such that $f=y_{1}^{k+1}+y_{2}^{2}+\ldots+y_{n}^{2}$. In statement 2 (ii) it is enough to check the case $m=k$, which reduces to the evident implication

$$
\left.g \in\left\langle f_{x_{1}}, \ldots, f_{x_{n}}, x_{n}\right\rangle \quad \Longrightarrow \quad g\right|_{L} \in\left\langle\left.\left(f_{x_{1}}\right)\right|_{L}, \ldots,\left.\left(f_{x_{n-1}}\right)\right|_{L}\right\rangle
$$

Statement 2(iii) is straightforward.

Now we are going to prove (64) by induction on $n$; the case $n=1$ is trivial, the case of $n=2$ is covered by Lemma 7 and Proposition 10. Assume that $n \geq 3$ and that (64) is proven for $n-1$. Let $(s-1)^{n}<k \leq s^{n}$. Put $m_{0}=s^{n-1}$. By the induction assumption there is a function germ $h=h\left(x_{1}, \ldots, x_{n-1}\right):(L, 0) \rightarrow(\mathbb{C}, 0)$ with an isolated critical point of type $A_{2 m_{0}-1}$ such that

$$
H^{1}\left(L, \mathcal{J}_{Z_{0}(h)}(p)\right)=0, \quad p \geq\left[b_{n-1} 2^{1 /(n-1)} s\right] .
$$

By a linear coordinate change in $L$ we can turn $h$ into

$$
h\left(x_{1}, \ldots, x_{n-1}\right)=x_{2}^{2}+\ldots+x_{n-1}^{2}+\text { h.o.t. }
$$

In particular, the system

$$
h_{x_{2}}=\ldots=h_{x_{n-1}}=0
$$

has a solution $x_{2}=x_{2}\left(x_{1}\right), \ldots, x_{n-1}=x_{n-1}\left(x_{1}\right)$ in a neighborhood of 0 , and

$$
\varphi\left(x_{1}\right)=h_{x_{1}}\left(x_{1}, x_{2}\left(x_{1}\right), \ldots, x_{n-1}\left(x_{1}\right)\right)=\alpha x_{1}^{2 m_{0}-1}+O\left(x_{1}^{2 m_{0}}\right), \quad \alpha \neq 0 .
$$

Define a function germ $f:\left(\mathbb{C}^{n}, 0\right) \rightarrow(\mathbb{C}, 0)$ by

$$
f\left(x_{1}, \ldots, x_{n}\right)=h\left(x_{1}, \ldots, x_{n-1}\right)+x_{n}^{2}-2 x_{n} \psi\left(x_{1}\right)
$$

with certain $\psi:(\mathbb{C}, 0) \rightarrow(\mathbb{C}, 0)$. Here the system

$$
f_{x_{2}}=\ldots=f_{x_{n}}=0
$$

defines a curve $\gamma:(\mathbb{C}, 0) \rightarrow\left(\mathbb{C}^{n}, 0\right)$

$$
x_{1}=t, x_{2}=x_{2}(t), \ldots, x_{n-1}=x_{n-1}(t), x_{n}=\psi(t),
$$

and we impose the condition $\left(\gamma \cdot f_{x_{1}}\right)_{0}=k$, saying that $f$ has an isolated critical point of type $A_{k}$. To satisfy the last condition, we choose $\psi$ so that

$$
f_{x_{1}}\left(t, x_{2}(t), \ldots, x_{n-1}(t), \psi(t)\right)=t^{k},
$$


which in view of $f_{x_{1}}=h_{x_{1}}-2 x_{n} \psi^{\prime}\left(x_{1}\right)$ reads as

$$
\varphi(t)-2 \psi(t) \psi^{\prime}(t)=t^{k}
$$

and is solved with respect to $\psi$ as follows:

$$
\begin{gathered}
\psi^{2}(t)=\int_{0}^{t}\left(\varphi(t)-t^{k}\right) d t=\frac{\alpha}{2 m_{0}} t^{2 m_{0}}+O\left(t^{2 m_{0}+1}\right)-\frac{t^{k+1}}{k+1} \\
\Longrightarrow \psi(t)=\sqrt{\frac{\alpha}{2 m_{0}}} t^{m_{0}}+O\left(t^{m_{0}+1}\right) .
\end{gathered}
$$

Observe also that the last formula means that $(\gamma \cdot L)_{0}=m_{0}$, and thus that Lemma 14 applies.

We complete the proof by establishing

$$
H^{1}\left(\mathcal{J}_{Z_{0}(f) / \mathbb{P}^{n}}\left(s_{0}+2 s\right)\right)=0, \quad s_{0}=\left[b_{n-1} 2^{1 /(n-1)} s\right] .
$$

Indeed, consider the exact sequences $\mathcal{E}_{i}, i=1, \ldots, 2 s$,

$$
\begin{gathered}
\mathcal{E}_{i}: \quad H^{1}\left(\mathcal{J}_{Z_{0}(f): L^{i} / \mathbb{P}^{n}}\left(s_{0}+2 s-i\right)\right) \rightarrow H^{1}\left(\mathcal{J}_{Z_{0}(f): L^{i-1} / \mathbb{P}^{n}}\left(s_{0}+2 s-i+1\right)\right) \\
\rightarrow H^{1}\left(L, \mathcal{J}_{\left(Z_{0}(f): L^{i-1}\right) \cap L}\left(s_{0}+2 s-i+1\right)\right) .
\end{gathered}
$$

By Lemma 14(2)(ii)

$$
\left(Z_{0}(f): L^{i-1}\right) \cap L \quad \subset \quad Z_{0}(f) \cap L \quad \subset \quad Z_{0}(h) ;
$$

hence the last term in all the sequences vanishes by 6 (65). Observing that $Z_{0}(f)$ : $L^{2 s}=\emptyset$ by Lemma 14(2)(iii) and $k / m_{0} \leq s$, we conclude that all terms in the sequence $\mathcal{E}_{2 s}$ vanish, and then moving inductively by decreasing $i$, we obtain that the middle term in $\mathcal{E}_{1}$, which appears in 65 , vanishes.

\section{ACKNOWLEDGEMENTS}

I would like to thank G.-M. Greuel and C. Lossen for very useful remarks and comments which allowed me to correct mistakes and improve the presentation.

\section{REFERENCES}

1. J. Alexander and A. Hirschowitz, An asymptotic vanishing theorem for generic unions of multiple points, Invent. Math. 140 (2000), no. 2, 303-325. MR 2001i:14024

2. C. Ciliberto and R. Miranda, The Segre and Harbourne-Hirschowitz conjectures, Applications of algebraic geometry to coding theory, physics and computation (Eilat, 2001), NATO Sci. Ser. II Math. Phys. Chem., vol. 36, Kluwer Acad. Publ., Dordrecht, 2001, pp. 37-51. MR 2002k:14011

3. E. D. Davis, 0-dimensional subschemes of $\mathbb{P}^{2}$ : New applications of Castelnuovo's function, Ann. Univ. Ferrara 32 (1986), 93-107. MR 89c:14009

4. S. Diaz and J. Harris, Ideals associated to deformations of singular plane curves, Trans. Amer. Math. Soc. 309 (1988), 433-468. MR 89m:14003

5. G.-M. Greuel and C. Lossen, Equianalytic and equisingular families of curves on surfaces, Manuscripta Math. 91 (1996), no. 3, 323-342. MR 98g:14023

6. G.-M. Greuel, C. Lossen, and E. Shustin, Plane curves of minimal degree with prescribed singularities, Invent. Math. 133 (1998), no. 3, 539-580. MR 99g:14035

7. G.-M. Greuel, C. Lossen, and E. Shustin, Castelnuovo function, zero-dimensional schemes and singular plane curves, J. Alg. Geom. 9 (2000), no. 4, 663-710. MR 2001g:14045

8. D. A. Gudkov and E. I. Shustin, On the intersection of the close algebraic curves, Topology (Leningrad, 1982), Lect. Notes Math., vol. 1060, Springer, Berlin, 1984, pp. 278-289. MR 86i: 14008 
9. S. M. Gusein-Zade and N. N. Nekhoroshev, On $A_{k}$-singularity on plane curves of fixed degree, Func. Anal. i Prilozhen. 34 (2000), no. 3, 69-70 (Russian) (English translation in Func. Anal. Appl. 34 (2000), 214-215). MR 2001k:14055

10. A. Hirano, Constructions of plane curves with cusps, Saitama Math. J. 10 (1992), 21-24. MR 94a:14029

11. F. Hirzebruch, Singularities of algebraic surfaces and characteristic numbers, Contemp. Math. 58 (1986), 141-155. MR 87j:14057

12. A. Hirschowitz, Une conjecture pour la cohomologie des diviseurs sur les surfaces rationelles génériques, J. Reine Angew. Math. 397 (1989), 208-213. MR 90g:14021

13. T. Keilen and I. Tyomkin, Existence of curves with prescribed topological singularities, Trans. Amer. Math. Soc. 354 (2002), 1837-1860. MR 2003a:14041

14. C. Lossen, New asymptotics for the existence of plane curves with prescribed singularities, Comm. Algebra 27 (1999), 3263-3282. MR 2000e:14037]

15. J. N. Mather, Stability of $C^{\infty}$-mappings, III: Finitely determined map-germs, Publ. Math. IHES 35 (1968), 127-156. MR 43:1215a

16. J. N. Mather and S.-T. Yau, Classification of isolated hypersurface singularities by their moduli algebras, Invent. Math. 69 (1982), 243-251. MR 84c:32007.

17. A. A. du Plessis and C. T. C. Wall, Singular hypersurfaces, versality and Gorenstein algebras, J. Alg. Geom 9 (2000), no. 2, 309-322. MR 2000k:14034

18. F. Severi, Vorlesungen über Algebraische Geometrie (Anhang F), Leipzig, Teubner, 1921.

19. E. Shustin, Real plane algebraic curves with prescribed singularities, Topology 32 (1993), 845-856. MR 95f:14049

20. E. Shustin, Geometry of equisingular families of plane algebraic curves, J. Alg. Geom. 5 (1996), no. 2, 209-234. MR 97g:14025

21. E. Shustin, Lower deformations of isolated hypersurface singularities, Algebra i Analiz 11 (1999), no. 5, 221-249 (English translation in St. Petersburg Math. J. 11 (2000), no. 5, 883908). MR 2000m:32039

22. B. L. van der Waerden, Einführung in die algebraische Geometrie, 2nd edition, Springer, Berlin, 1973. MR 49:8984

23. J. Wahl, Equisingular deformations of plane algebroid curves, Trans. Amer. Math. Soc. 193 (1974), 143-170. MR 54:7460

24. G. Xu, Curves in $\mathbb{P}^{2}$ and symplectic packings, Math. Ann. 299 (1994), 609-613. MR 95f:14058

25. G. Xu, Ample line bundles on smooth surfaces, J. Reine Angew. Math. 469 (1995), 199-209. MR 96k:14003

School of Mathematical Sciences, Raymond and Beverly Sackler Faculty of Exact Sciences, Tel Aviv University, Ramat Aviv, 69978 Tel Aviv, Israel

E-mail address: shustin@post.tau.ac.il 